\title{
Animal Cognition \\ Comparing supervised learning methods for classifying sex, age, context and individual Mudi dogs from barking \\ --Manuscript Draft--
}

\begin{tabular}{|c|c|}
\hline Manuscript Number: & ANCO-D-13-0010 \\
\hline Full Title: & $\begin{array}{l}\text { Comparing super } \\
\text { Mudi dogs from ba }\end{array}$ \\
\hline Article Type: & Original Paper \\
\hline Corresponding Author: & $\begin{array}{l}\text { Pedro Larranaga } \\
\text { SPAIN }\end{array}$ \\
\hline \multicolumn{2}{|l|}{$\begin{array}{l}\text { Corresponding Author Secondary } \\
\text { Information: }\end{array}$} \\
\hline \multicolumn{2}{|l|}{ Corresponding Author's Institution: } \\
\hline \multicolumn{2}{|l|}{$\begin{array}{l}\text { Corresponding Author's Secondary } \\
\text { Institution: }\end{array}$} \\
\hline First Author: & Ana Larranaga \\
\hline \multicolumn{2}{|l|}{ First Author Secondary Information: } \\
\hline \multirow[t]{6}{*}{ Order of Authors: } & Ana Larranaga \\
\hline & Concha Bielza \\
\hline & Peter Pongracz \\
\hline & Tamas Farago \\
\hline & Anna Balint \\
\hline & Pedro Larranaga \\
\hline
\end{tabular}

Order of Authors Secondary Information:

Abstract:

Barking is perhaps the most characteristic form of vocalization in dogs, however, very little is known about its role in the intra-specific communication of this species. Besides the obvious need for ethological research, both in the field and in the laboratory, the possible information content of barks can also be explored by computerized acoustic analyses. This study compares four different supervised learning methods (naive Bayes, classification trees, k-nearest neighbors and logistic regression) combined with three strategies for selecting variables (all variables, filter and wrapper feature subset selections) to classify Mudi dogs by sex, age, context and individual from their barks. The classification accuracy of the models obtained was estimated by means of K-fold cross-validation. Percentages of correct classifications were $85.13 \%$ for determining sex, $80.25 \%$ for predicting age (recodified as young,

15 adult and old), $55.50 \%$ for classifying contexts (seven situations) and $67.63 \%$ for recognizing individuals ( 8 dogs), so the results are encouraging. The best performing method was k-nearest neighbors following a wrapper feature selection approach. The results for classifying contexts and recognizing individual dogs were better with this method than they were for other approaches reported in the specialized literature. This is the first time that the sex and age of domestic dogs have been predicted with the help of sound analysis. This study shows that dog barks carry ample information regarding the caller's indexical features. Our computerized analysis provides indirect proof that barks may serve as important source of information for dogs as well.

Response to Reviewers:

No comments 
Animal Cognition manuscript No.

(will be inserted by the editor)

\title{
Comparing supervised learning methods for classifying sex, age, context and individual Mudi dogs from barking
}

\author{
Ana Larrañaga · Concha Bielza · Péter Pongrácz · Tamás \\ Faragó · Anna Bálint · Pedro Larrañaga
}

Received: date / Accepted: date

\begin{abstract}
Barking is perhaps the most characteristic form of vocalization in dogs, however, very little is known about its role in the intra-specific communication of this species. Besides the obvious need for ethological research, both in the field and in the laboratory, the possible information content of barks can also be explored by computerized acoustic analyses. This study compares four different supervised learning methods (naive Bayes, classification trees, $k$-nearest neighbors and logistic regression) combined with three strategies for selecting variables (all variables, filter and wrapper feature subset selections) to classify Mudi dogs by sex, age, context and individual from their barks. The classification accuracy of the models obtained was estimated by means of $K$-fold cross-validation. Percentages of correct classifications were $85.13 \%$ for determining sex, $80.25 \%$ for predicting age (recodified as young, adult and old), $55.50 \%$ for classifying contexts (seven situations) and $67.63 \%$ for recognizing individuals ( 8 dogs), so the results
\end{abstract}

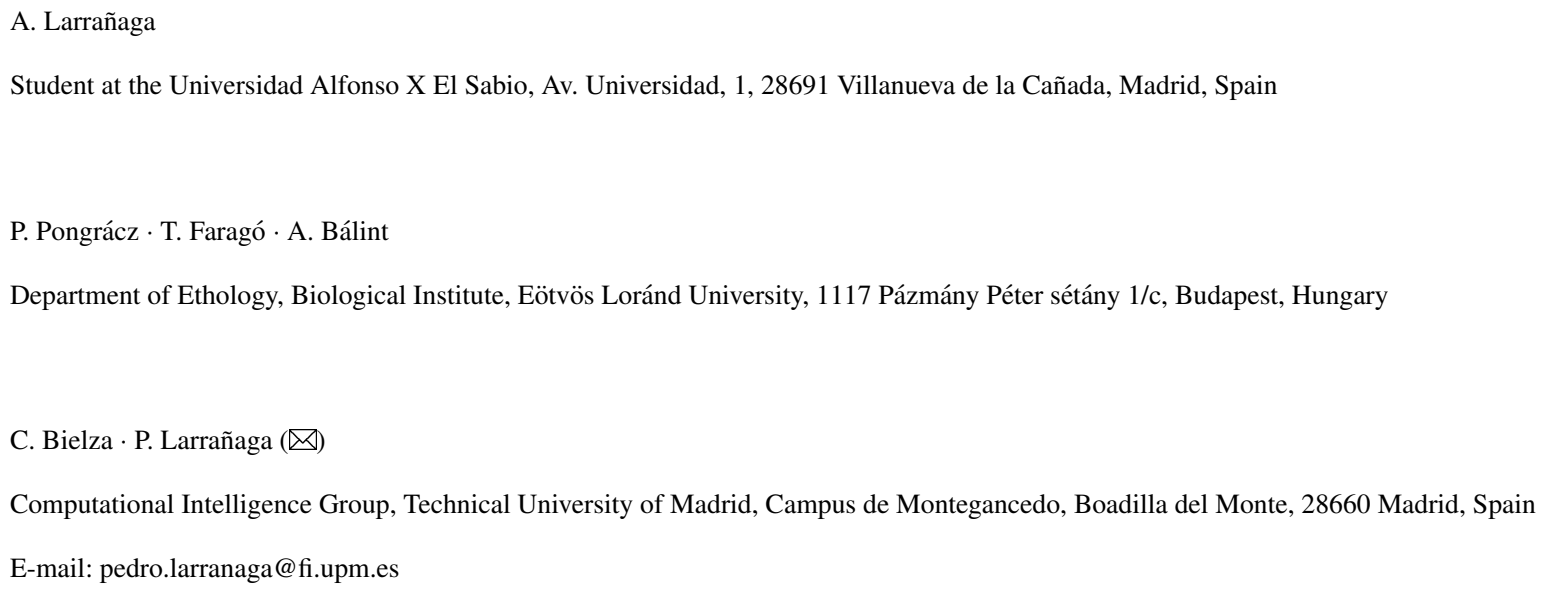


are encouraging. The best performing method was $k$-nearest neighbors following a wrapper feature selection approach. The results for classifying contexts and recognizing individual dogs were better with this method than they were for other approaches reported in the specialized literature. This is the first time that the sex and age of domestic dogs have been predicted with the help of sound analysis. This study shows that dog barks carry ample information regarding the caller's indexical features. Our computerized analysis provides indirect proof that barks may serve as an important source of information for dogs as well.

Keywords Mudi dog barks · Acoustic communication $\cdot$ Feature subset selection $\cdot$ Machine learning $\cdot$ Supervised classification $\cdot K$-fold cross-validation

\section{Introduction}

Canine communication (including dog-human communication) has become a well studied topic among ethologists in the last decade. Most efforts have focused on how and to what extent dogs are able to understand different forms of human communication, through visual gestures (Reid 2009), voice recognition (Adachi et al 2007), acoustic signals for ceasing or intensifying their activity (McConnell and Baylis 1985; McConnell 1990), and ostensive signals (Téglás et al 2012). However, it has also been found that dogs can get their message across to humans, for example by turning their head or alternating their gaze between the human and their target (Miklósi et al 2000), and that dogs can emulate other behavioral forms so as to convey feelings, of guilt for example, in an appropriate situation (Hecht et al 2012).

Unlike taxon-specific chemical and visual communication (Meints et al 2010; Wan et al 2012), acoustic signals are regarded as highly conservative and uniformly constructed within such broad groups of animals as avian and mammalian species. Morton (1977) provided a set of so-called 'motivation-structural' rules to explain this point. According to his theory, the quality of the sound (pitch, tonality) strongly depends on the physical (anatomical) constraints of the animal's voice-producing tract, which in turn depends on the physical features of the animal itself (size, for example). Stronger, larger specimens within a species will usually be the dominant, aggressive animals and smaller, younger individuals are usually the subordinates. Thus, the typical vocalizations (low pitched, broadband, noisy) emitted by the larger, more aggressive individuals, for example, could, according to Morton, evolve into the trademarks of agonistic 
inner states. Similarly, the typical vocal features of a smaller, subordinate animal (high pitched, narrow band, tonal) could project the lack of aggressive intent communicative meaning.

Dogs have a rich vocal repertoire, see (Cohen and Fox 1976; Tembrock 1976; Yeon 2007), like other closely related wild members of the Canidae family. The ethological analysis of the possible functions of canine vocalizations has so far provided data about the individual-specific content of wolf howls (Mazzini et al 2013; Root-Gutteridge et al 2013), the indexical content of dog growls, related to the caller's body size (Taylor et al 2008, 2010; Faragó et al 2010a; Bálint et al 2013), and the context-specific content of dog growls (Faragó et al 2010b; Taylor et al 2009). However, even though barking is considered to be the most characteristic form of dog vocalization, exceeding the barks of wolves and coyotes both in its frequency of occurrence and variability (Cohen and Fox 1976), the functional aspects of dog barks are surprisingly little known. The theoretical framework for the information content and evolution of barking in the dog involves very different assumptions, ranging from the theory that it is a non-communicative byproduct of domestication (Coppinger and Feinstein 1991), through the low-information level mobbing signal theory (Lord et al 2000), to the context-specific information source theory (Feddersen-Petersen 2000; Yin 2002; Pongrácz et al 2010). As dogs are the oldest domesticated companions of humans (Druzhkova et al 2013), dog barking may have acquired a 'new target audience' in humans during the many thousand years of co-existence. A possible indirect proof of this is a series of playback experiments which showed that humans are able to correctly categorize barks according to their contexts (Pongrácz et al 2005). As for contextual content, human listeners also had consistent opinions about the inner state of the barking dogs, and the acoustic analysis of the barks revealed that humans base their decision on the kinds of acoustic parameters of the barks that were expected on the basis of Morton's theory (Pongrácz et al 2006). Besides the pitch and the harmonic-to-noise ratio, however, it was found that the inter-bark interval (or 'pulsing') of the barks is also important when assessing the inner state of the barking dog.

Although there are convincing empirical demonstrations that dog barks show acoustic features that are seemingly context-specific (Yin 2002; Pongrácz et al 2005), and we have also learned that humans can decipher information from dog barks regarding the context of vocalization and the inner state of the animal, it is less well understood whether dog barks carry an equally rich (or even richer) content of information for another dog. Until now, there have been only a few experiments with dogs as subjects which revealed that dog barks do carry individual-specific cues. One used a habituation-dishabituation paradigm (Maros et al 2008; Molnár et al 2009), and the other was a computerized 
bark analysis study (Molnár et al 2008). These results raise the question of whether dog barks carry a much wider set of information about the vocalizing animal than humans are able to decipher. Another intriguing problem is which acoustic parameters could be responsible for the finer details of the information content of dog barks. Based on the vast literature of vocalization-based sex and individual recognition in other species, e.g., African wild dog, Lycaon pictus (Hartwig 2005); white-faced whistling duck, Dendrocygna viduata (Volodin et al 2005); or Wied's black-tufted-ear marmosets, Callithrix kuhlii (Smith et al 2009), one might expect dog barks to also carry specific cues of the caller's individual features, such as sex and age, for example. There are, however, considerable obstacles in testing such subtle pieces of information using classical techniques (i.e. playback). Fortunately, the current age of computer-based methods opens up the possibility for analyzing and testing lots of sound samples with the help of artificial intelligence.

Machine learning techniques have been used in behavioral research on acoustic signals for a wide range of species, see Table 1. For dolphins, artificial neural networks have been applied to model dolphin sonar, specifically for discriminating differences in the wall thickness of cylinders using time and frequency information from the echoes (Au et al 1995). Also, support vector machines and quadratic discriminant function analysis have been used to classify fish species according to their echoes using a dolphin-emulating sonar system (Yovel and Au 2010), and Gaussian mixture models and support vector machines have been employed to classify echolocation clicks from three species of odontocetes (Roch et al 2008). Differentiation of categories or graded barks in mother-calf vocal communication in Atlantic walrus have been analyzed with artificial neural networks and discriminant functions (Charrier et al 2010). Frog song identification to recognize frog species has been carried out with $k$-nearest neighbor classifiers and support vector machines (Hunag et al 2009). Linear discriminant analysis, decision tree, and support vector machines have been employed to automate the classification of calls of several frog and bird species (Acevedo et al 2009). Gaussian mixture models have also been used for individual animal recognition in birds (Cheng et al 2010). Bat species have been acoustically identified using artificial neural networks (Parsons 2001; Britzke et al 2011), discriminant function analysis (Parsons and Jones 2000; Britzke et al 2011), classification trees (Adams et al 2010), $k$-nearest neighbors (Britzke et al 2011) as well as other classifiers (random forests and support vector machines) whose behavior has been compared (Armitage and Ober 2010). Artificial neural networks have been used to discriminate between the sounds of different animals within a group of British insect species (Orthoptera), including crickets and grasshoppers (Chesmore 2001). Blumstein and Munos (2005) found potentially significant information about identity, age and sex encoded in 
yellow-bellied marmots calls using discriminant function analysis. For suricates, discriminant function analysis was chosen to predict the predator type (mammal, bird and snake) from the alarm calls (Manser et al 2002). Hidden Markov models have been used to analyze African elephant vocalizations and speaker identification, discrimination of rumbles in different contexts, and oestrous cycle phase determination from rumbles of female elephants (Clemins 2005). Moreover, other work has focused on identifying calls from different animals such as bears, eagles, elephants, gorillas, lions and wolves, with $k$-nearest neighbor classifiers, artificial neural networks and hybrid methods (Gunasekaran and Revathy 2011).

Table 1 about here.

For canids, research analyzing the acoustic measures of barks with machine learning methods is limited, see Table 1. Discriminant functions have been used for individual recognition within a wild population of Arctic foxes (Frommolt et al 2003), and African wild dogs (Hartwig 2005). Domestic dog barks have been analyzed again using discriminant analysis (Yin and McCowan 2004) for classification into context-based subtypes (three different contexts) and in order to identify individual dogs. These two tasks were further refined in the same paper to categorize each individual's barks into separate contexts and identify the individual barking within each context. A total of 4,672 barks were recorded from ten dogs of six different breeds and 120 variables were extracted from the spectrograms. More recently, 6,006 barks of 14 Mudi breed individuals were recorded under six different communicative situations (Molnár et al 2008). After processing the spectrograms of their signals a genetic programming-based heuristic guided the construction of new descriptors. The aims were the same as in Yin and McCowan (2004), although the machine learning technique was a Gaussian naive Bayes classifier.

In this paper we extend Molnár et al.'s research in several ways. As in Molnár et al (2008), we classify barks into contexts and identify individual barks. Unlike Molnár et al., we also investigate whether barks encode information about dog sex and age. Also, we specify context classification per individual dog and recognize individual bark per context. Therefore we have six different classification problems concerning sex, age, contexts, contexts per individual, individuals and individuals per context. Moreover, for each of these six problems, a thorough set of four machine learning models (Gaussian naive Bayes, classification trees, $k$-nearest neighbors and logistic regression) are trained from a 
database of 800 barks corresponding to 8 Mudi dogs in seven behavioral contexts. Their performance is estimated using cross-validation ( $K$-fold scheme) which assesses the ability to classify barks that had not been previously encountered. Given an incoming Mudi dog bark, two models (Gaussian naive Bayes and logistic regression) output the probability of each class value, whereas the other two models deterministically provide the predicted class value. Gaussian naive Bayes assumes normality and independence of the features given the class value. Logistic regression uses the sigmoid function of a linear combination of the features as the probability of each class value. Classification trees hierarchically partition the feature space. Finally, $k$-nearest neighbors simply predicts the class value by majority voting in a feature space neighborhood. The diversity of these four models are representative of the available supervised classifiers. Rather than using all the extracted acoustic measures, we selected relevant features with two methods, filter and wrapper, for each machine learning model. Whereas wrapper methods use a predictive model to score feature subsets, filter methods use a proxy measure instead of the classification accuracy to score the selected features.

\section{Methods}

Subjects

Barks recorded from Mudi dogs were used for this study. The Mudi is a medium-sized Hungarian herding dog breed. The Mudi breed standard is listed as \#238 with the FCI (Fédération Cynologique Internationale). Initially we collected 7,310 barks from 27 individuals. The number of barks per dog ranged from 8 to 1,696. These barks were recorded in different number of bouts for each dog. Trying to minimize the effect of pseudoreplication we only considered dogs whose initial number of barks was greater than 300. From each of these 8 dogs, 100 barks were randomly selected using a systematic sampling procedure, thereby balancing the number of samples coming from each individual. Table 2 contains the characteristics of these selected 800 barks according to sex ratio (male/female: 3/5), age (ranging from 1 year to 10 years old), number of bouts for each dog (with a minimum of 5 and a maximum of 14) and number of barks per dog in each of the seven contexts. Age values are grouped into intervals to form a three-valued class variable: young dogs (1-3 years old), adult dogs (4-8 years old) and old dogs (more than 8 years old).

Table 2 about here. 
Recording and processing of the sound material

\section{Recording contexts}

Recordings were made using a Sony TCD-100 DAT tape recorder and Sony ECM-MS907 microphone on Sony PDP65C DAT tapes. During recording of the barks, the experimenter held the microphone at a distance of 3 to 4 meters from the dog. We collected bark recordings in seven different behavioral contexts. With the exception of two contexts (Alone and Fight), all recordings were done at the dog's residence. Barks of the Fight context were recorded at dog training schools. The training school dogs were also taken to a park or other suitable outdoor area to record the Alone barks. The seven situations are as follows:

- Alone ( $N=106$ recordings): The owner and the experimenter (male, 23 years old) took the dog to a park or other outdoor area, where the dog was tied to a tree or fence by its leash. The owner left the dog and walked out of the dog's sight, while the experimenter remained with the dog and recorded its barks.

- Ball $(N=131)$ : The owner held a ball (or one of the dog's favorite toys) approximately $1.5 \mathrm{~m}$ in front of the dog.

- Fight $(N=131)$ : For dogs to perform in this situation, the trainer acts as if he intends to attack the dog-owner dyad. Dogs are expected to bark aggressively and even bite the trainer's glove. The owner keeps the dog on a leash during this exercise.

- Food $(N=106)$ : The owner held the dog's food bowl approximately $1.5 \mathrm{~m}$ in front of the dog.

- Play $(N=89)$ : The owner was asked to play a game with the dog, such as tug-of-war, chasing or wrestling. The experimenter recorded the barks emitted during this interaction.

- Stranger $(N=206)$ : The experimenter acted as the 'stranger' for all the dogs, and appeared at the dog owners' garden or front door. The experimenter recorded the barking dog for 2-3 minutes. The owner was not in the vicinity (in the garden, or near to the entrance) during the recording.

- Walk $(N=31)$ : We asked the owner to behave as if he/she was preparing to go for a walk with the dog. For example, the owner took the dog's leash in her/his hand and told the dog "We are leaving now". 
Initial processing of the sound material

The recorded material was digitalized with a 16-bit quantization and $44.10 \mathrm{kHz}$ sampling rate using a TerraTec DMX 6Wre 24/96 sound card. As each recording could contain at least three or four barks, individual bark sounds were manually segmented and extracted. This process resulted in a final collection of 7,310 sound files containing only a single bark sound. Obviously these sounds are not independent from a statistical point of view. As some of the machine learning methods used in this work assume that the samples are independent and identically distributed, we randomly selected non-consecutive barks, alleviating in this way the pseudoreplication effect. The final data set contains 800 barks from the initial 7,310 sound files.

Sound analysis

Based on the initial parameter set used in Molnár et al (2008), 29 acoustic measures were extracted from the bark samples with an automated Praat script, see Table 3 and Figure 1.

Table 3 about here.

Figure 1 about here.

The energy, loudness and the long-term average spectrum (LTAS) are measurements of sound energy, and the LTAS parameters reflect its change over time, whereas the spectral parameters show the distribution of energy over the frequency components.

According to the Source-Filter framework (Fant 1976), the fundamental frequency is the lowest harmonic component of the source signal that is produced in the larynx by the movements of the vocal fold. Measurements of the fundamental show the modulation of this source signal over time. One voice cycle is the unit of the movements of the vocal folds. During sound production the repeated opening and closing of the vocal folds generates cyclic pressure changes in the exhaled air, which will be the sound wave itself. Measurements of the vocal cycles show the regularities in voice production. 
Finally, tonality or harmonics-to-noise ratio gives the proportion of regular, tonal frequency components over the noise caused by the irregular movements of the vocal folds, or the turbulences in the air flow in the vocal tract. These measurements are capable of describing the quality of the sound and its change over time.

The process is illustrated in Figure 2 (top).

Figure 2 about here.

Supervised classification

A common machine learning task is pattern recognition (Duda et al 2001), in which two different problems are considered depending on the available information. We always started from a data set in which each case or instance (a single bark sound in this paper) is characterized by features or variables (29 acoustical measures in our case). In a supervised classification problem, an additional variable - called the class variable - contains the instance label (sex, age, context or individual in this paper) and we look for a model able to predict the label of a new case with known features. Alternatively, in an unsupervised classification problem or clustering (Jain et al 1999), the label is missing and the aim is to form groups or clusters with cases (dog barks) that are similar with respect to the features at hand.

In this paper we apply supervised classification methods to automatically learn models from data. These models will be used to separately predict dog sex, dog age, context, and the individual dog, from a set of predictor variables capturing the acoustical measures of the dog barks.

In a binary supervised classification problem, there is a feature vector $\mathbf{X} \in \mathbb{R}^{n}$ whose components, $X_{1}, \ldots, X_{n}$, are called predictor variables and there is also a label or class variable $C$ taking values on $\{0,1\}$. The task is to induce classifier models from training data, which consists of a set of $N$ observations $\mathscr{D}_{N}=\left\{\left(\mathbf{x}^{(1)}, c^{(1)}\right), \ldots,\left(\mathbf{x}^{(N)}, c^{(N)}\right)\right\}$ drawn from the joint probability distribution $p(\mathbf{x}, c)$, see Table 4 . In our dog data set, $n=8$ acoustical measures and $N=800$ bark sounds. The classification model will be used to assign labels to new instances, $\mathbf{x}^{(N+1)}$, only characterized by the values of the predictor variables.

Table 4 about here.

To quantify the goodness of a binary classification model, true positives $(T P)$, true negatives $(T N)$, false positives $(F P)$ and false negatives $(F N)$ are counted over the test data and placed in a confusion matrix. This confusion 
matrix contains in its diagonal the $T P$ and $T N$ observations. Then we can define the error rate as $\frac{|F N|+|F P|}{N}$, where $N=|T P|+|F P|+|T N|+|F N|$ is the total number of instances, or equivalently, the accuracy as $\frac{|T P|+|T N|}{N}$.

Dog sex classification is binary, $\Omega_{C}=\{$ Female, Male $\}$, where there are two possible errors: predict a Male as a Female dog, and alternatively predict a Female as a Male.

The other classifications are multiclass, where $C$ takes $r>2$ possible class values. Let $\Omega_{C}=\{1,2, \ldots, r\}$ denote this set. Thus, $\Omega_{C}=\{$ Young, Adult, Old $\}$ for age, $\Omega_{C}=\{$ Alone, Ball, Fight, Food, Play, Stranger, Walk $\}$ for contexts, and $\Omega_{C}=\{\operatorname{dog} 1, \ldots, \operatorname{dog} 8\}$ for individuals in our case. The $r \times r$-dimensional confusion matrix contains all pairwise counts, $m_{i j}$, the number of cases out of $N$ from the real class $c_{i}$ classified by the model as $c_{j}$. The accuracy is given by $\sum_{i=1}^{r} m_{i i} / N$

Accuracy estimation of supervised classification models

An important issue is how to honestly estimate the (expected) accuracy of a classification model when using this model for classifying unseen (new) instances. A simple method is to partition the whole data set into two subsets: the training subset and the test subset. According to this training and testing scheme, the classification model is learned from the training subset, and it is then used in the test subset for the purpose of estimating its accuracy. However, the information in the data set is under-used, as the classification model is learned from a subset of the original data set.

In this paper we will use an estimation method called $K$-fold cross-validation (Stone 1974). This uses the whole data set to honestly learn the model. The data set is partitioned into $K$ folds of approximately the same size. Each fold is left out of the learning process, which is carried out with the remaining $K-1$ folds, and used later as a test set. This process is repeated $K$ times. Thus, every instance is in a test set exactly once and in a training set $K-1$ times. The model accuracy is estimated as the mean of the accuracies for each of the $K$ test sets. In our experiments we will fix the value of $K$ to 10 . 
Feature subset selection

The feature subset selection (FSS) problem (Liu and Motoda 1998) refers to the question of whether all the $n$ predictor features are really useful for classifying the instances with a given model. The FSS problem can be formulated as follows: given a set of candidate features, select the best subset under some classification learning method.

This dimensionality reduction by means of an FSS process has several potential advantages for a supervised classification model, such as the reduction in the cost of data acquisition, an improved understanding of the final classification model, a faster induction of the classification model, and an improvement in classifier accuracy.

FSS can be viewed as a search problem, where each state in the search space specifies a subset of selectable features. An exhaustive search of all possible feature subsets, given by $2^{n}$, is usually unfeasible in practice because of the large computational burden, and heuristic search is usually used.

For a categorization of FSS see Saeys et al (2007). There are two main types of FSS depending on the function used to measure the goodness of each selected subset. In the wrapper approach to the FSS, the accuracy reported by a classifier guides the search for a good subset of features. We have used a greedy stepwise search in our experiments, i.e. one that progresses forward from the empty set selecting at each step the best option among adding a variable not yet included within the model and deleting a variable from the current model. The search is halted when neither of these options improves model accuracy. When the learning algorithm is not used in the evaluation function, the goodness of a feature subset can be assessed using only intrinsic data properties, such as an information theory based evaluation function. This is the filter approach to the FSS problem. In this paper we apply both wrapper and filter approaches to the FSS problem. For the second type, a multivariate filter based on mutual information, called correlation feature selection, is used (Hall 1999). This tries both to minimize redundancy between selected features and maximize correlation with the class variable.

\section{Supervised classification methods}

Given an instance $\mathbf{x}$, supervised classification builds a function $\gamma$ that assigns to $\mathbf{x}$ a class label in $\Omega_{C}=\{1, \ldots, r\}$. We provide a short description of each supervised classification method used. 
Naive Bayes (Minsky 1961) is the simplest Bayesian classifier. A Bayesian classifier assigns the most probable $a$ posteriori class to a given instance $\mathbf{x}$, i.e. it yields the $c$ value of $C$ that maximizes the posterior probability $p(c \mid \mathbf{x})$. Using the Bayes' theorem, this is equivalent to maximizing $p(c) p(\mathbf{x} \mid c)$. The naive Bayes is built upon the assumption of conditional independence of the predictive variables given the class. Computationally this means that $p(\mathbf{x} \mid c)$ in the previous product is easily obtained as the product of all factors $p\left(x_{j} \mid c\right), j=1, \ldots, n$, each associated with one variable. The Gaussian naive Bayes classifier applies for continuous variables $X_{j}$ following a Gaussian distribution $f_{j}$. Therefore this model computes $c$ such that

$$
\max _{c \in \Omega_{C}} p(c) \prod_{j=1}^{n} f_{j}\left(x_{j} \mid c\right)
$$

In a classification tree (Quinlan 1993), the learned function $\gamma$ is represented by a decision tree. Each (non-leaf) node specifies a value test of some variable of the instance. Each descendant branch corresponds to one of the possible values for this variable. Each leaf node provides the class label given the values of the variables jointly represented by the path from the root to that leaf. Unseen instances are classified by sorting down the tree from the root to some leaf node testing the variable specified at each node. A classification tree is learned in a top-down manner (starting with the root node) by progressively splitting the training data set into smaller and smaller subsets based on variable value tests. This process is repeated on each derived subset in a recursive manner called recursive partitioning of the space representing the predictive variables. Key decisions are how to select which variable to test at each node in the tree, and how deep the tree should be, i.e. whether to stop splitting or select another variable and grow the tree further. These decisions make the differences between algorithms. The $\mathrm{C} 4.5$ algorithm used in this paper chooses variables by maximizing the gain ratio, which is the ratio of the information gain of $X_{j}$ and $C$ and the entropy of $X_{j}$, which are both concepts used in information theory. The algorithm incorporates post-pruning rules to avoid the tree becoming too deep thereby escaping from the training data overfitting, i.e. its failure to work well with new unseen instances.

The $k$-nearest neighbor classifier (Fix and Hodges 1951) is a non-parametric method that assigns to a given instance $\mathbf{x}$ the class label most frequently found amongst its $k$ nearest instances, that is, the predicted class is decided by examining the labels of the $k$ nearest neighbors and voting. A common distance used for obtaining the $k$ nearest neighbors for a continuous variable $\mathbf{x}$ is the Euclidean distance. This classifier is a type of lazy learning where the function is only approximated locally and all computation is deferred until classification. In our experiments we will fix $k=1$. 
Logistic regression (Le Cessie and van Houwelingen 1992), like naive Bayes, produces a posterior probability $p(c \mid \mathbf{x})$ for a given instance $\mathbf{x}$. For binary classification, the model assumes that it is a transformation of a linear combination of the input variables, given by

$$
p(C=1 \mid \mathbf{x})=1 /\left[1+e^{-\left(\beta_{0}+\beta_{1} x_{1}+\cdots+\beta_{n} x_{n}\right)}\right],
$$

where $\beta_{0}, \beta_{1}, \ldots, \beta_{n}$ are model parameters estimated from data by maximum likelihood. If $\mathscr{L}\left(\beta_{0}, \ldots, \beta_{n}\right)$ denotes the $\log$-likelihood function of the data under this model, the problem is to find $\beta$ s that maximize this function. The ridge logistic regression used in this paper adds a penalization term to $\mathscr{L}$, and the problem is then to maximize the function $\mathscr{L}\left(\beta_{0}, \ldots, \beta_{n}\right)-\lambda \sum_{j=1}^{n} \beta_{j}^{2}$, for $\beta$ s where $\lambda>0$ controls the amount of penalization. This penalty forces the parameters to shrink to zero achieving a reduction in the variance of the parameter estimates with an overall increased accuracy. For multiclass classification, the posterior probability of $c \neq r$ is given by

$$
p(c \mid \mathbf{x})=\frac{e^{\left(\beta_{0}^{(c)}+\beta_{1}^{(c)} x_{1}+\cdots+\beta_{n}^{(c)} x_{n}\right)}}{1+\sum_{l=1}^{r-1} e^{\left(\beta_{0}^{(l)}+\beta_{1}^{(l)} x_{1}+\cdots+\beta_{n}^{(l)} x_{n}\right)}}, \quad l=1, \ldots, r-1
$$

and hence $p(r \mid \mathbf{x})$ is derived from the others since they all sum to one. Note that in this multiclass case, we need a set of $n+1$ parameters $\left\{\beta_{0}^{(l)}, \beta_{1}^{(l)}, \ldots, \beta_{n}^{(l)}\right\}$ for each $l$ value, $l=1, \ldots, r-1$, that is, a total of $(n+1)(r-1)$ parameters.

All the results were calculated using WEKA software (Hall et al 2009).

\section{Results}

The six problems we will deal with are illustrated in Figure 2 (bottom).

Sex

The $k$-nearest neighbor classifier produced the best results, with an accuracy of $85.13 \%$, with a wrapper feature selection (in bold), see Table 5. This model contains 12 predictor variables, see Table 16. The groups that record spectral energy and source signal variables are under-represented, according to the categorization of acoustic measures provided in Table 3.

Table 5 about here. 

for males, $24.00 \%$ (72 false females from a total of 300 real males).

Table 6 shows the accuracies per dog of the $k$-nearest neighbor model with 12 predictors. The model accuracy when predicting the five female dogs is around $90 \%$, with the worst predictions for $\operatorname{dog} 3$ and $\operatorname{dog} 4(87.00 \%)$, and the best for $\operatorname{dog} 5(97.00 \%)$. The three male dogs are predicted with accuracies ranging from $73.00 \%$ for dog 1 to $79.41 \%$ for $\operatorname{dog} 7$.

Table 6 about here.

Supplementary Material contains the specifications of the best models for the prediction of the dog sex. For naive Bayes the univariate conditional Gaussian densities for each predictor variable are shown. The structure of the classification tree model is also presented, as well as the coefficients of the logistic regression model. For the $k$-nearest neighbor classifier the data set constitutes the model and therefore it is not shown.

Age

Table 7 (left) shows the age results. As for the sex prediction problem, $k$-nearest neighbors with a wrapper feature selection produced the best accuracy $80.25 \%$. The 15 selected variables in this model mainly contain measurements of spectral energy, sound energy and voice cycles. For this problem, the wrapper strategy outperformed the other strategies in the four supervised classification methods.

Table 7 about here.

The confusion matrix in Table 7 (right) of the best model shows that a Young dog is classified as Old in only 2.67\% of cases (8 out of 300), while old dogs are misclassified as Young in $6.86 \%$ of cases (7 out of 102). The error rates classifying Young, Adult and Old dogs are $21.00 \%, 17.59 \%$ and $24.51 \%$ respectively. These figures suggest that it is easier to get it wrong when classifying Young and Old dogs.

Table 8 contains the accuracies per dog of the best model. This model provides a $79.00 \%$ of accuracy when predicting Young dogs. This percentage is very similar for each of the three young $\operatorname{dog}(\operatorname{dog} 1, \operatorname{dog} 2 \operatorname{and} \operatorname{dog} 3)$. However for the four adult dogs the model shows a wide range of accuracies, varying from $66.00 \%$ (dog6) to $90.00 \%$ (dog5). Dog7, that is the only old dog, is classified with an accuracy of $75.49 \%$. 
Table 8 about here.

Supplementary Material contains the specifications of the best models for the prediction of the dog age.

Context

A single model for all dogs. Table 9 (left) shows the results of a single model learned from the 800 barks to discriminate among the 7 contexts: Alone, Ball, Fight, Food, Play, Stranger and Walk.

Table 9 about here.

$k$-nearest neighbor classifier and wrapper selection is once more the best-performing model with an accuracy of $55.50 \%$. The variables selected by this model correspond mainly to spectral energy and voice cycle measurements. Note that now we have a more difficult problem with more class values to be predicted ( 7 contexts) and consequently the estimated accuracy is expected to be lower.

From Table 9 (right) we can compute the contexts with the highest and lowest true positive rates, that correspond to Fight (0.76) and Walk (0.35), respectively. The Ball context is often misclassified as Food and vice versa. The same holds for the Walk and Play pair. This is quite reasonable since both pairs define quite similar underlying concepts. Many barks under Fight or Alone situations are misclassified as Stranger. However the Stranger context is usually confused with the Ball and Food context.

Table 10 contains the accuracies per dog of the best model. This model provides $43.40 \%$ accuracy when predicting the Alone context, with extreme prediction accuracies for $\operatorname{dog} 7$ (52.94\%) and $\operatorname{dog} 8(14.29 \%)$. The Ball context achieves $48.85 \%$ accuracy, having $\operatorname{dog} 7$ and $\operatorname{dog} 8$ the worst $(29.41 \%)$ and best $(64.29 \%)$ predictions, respectively. These two dogs also present the worst and best predictions for the Food context. The model shows better accuracies for the Fight and Stranger contexts. In the Fight context the $98.00 \%$ of success for dog 5 is noteworthy, whereas the worst behavior in the Stranger context is for $\operatorname{dog} 7(35.29 \%)$. The Play and Walk contexts show highly variable accuracies for the different dogs.

Table 10 about here.

Supplementary Material contains the specifications of the best models for the prediction of the dog context. 
A model per dog. More refined dog-specific models are built here. By selecting instances from the same dog, the corresponding model will identify the context for that dog. A total of 96 models ( 8 dogs $\times 12$ models per dog) have been considered, where only the performance of the best model is shown in Table 11.

Naive Bayes was the best model 3 times, $k$-nearest neighbors 4 times, and logistic regression in 2 cases. Regarding the feature subset selection methods, wrapper reports the best results for all 8 dogs.

Table 11 about here.

Table 11 shows that accuracies decrease in proportion to the increase in the number of contexts. With two contexts accuracies fall in the interval $[78 \%, 100 \%]$. The accuracies for the two dogs with four contexts are $74 \%$ and 73\%.Increasing the number of contexts to six and seven the accuracies are $59.80 \%$ and $66.98 \%$ respectively.

Figure 3 displays, for the best models in Table 11, the mean number of selected variables by the five types of acoustic variables. Spectral energy and voice cycle measurements were the two groups with more often selected (in relative terms) variables regardless of the number of barks.

Figure 3 about here.

From the previous table we select some models for the sake of illustration. Figure 4 shows the naive Bayes model which performed best for dog5, with only two observed contexts, Fight and Strange (see the first row in Table 11). The model is built with only five variables, Deviationfreq, Pitchmax, Pitchmaxt, Pitchd and Ppp selected by the wrapper approach. The missing arcs between predictor variables and the arcs from the class to the predictor variables encode the assumption of conditional independence underlying naive Bayes. Figure 4 also shows the parameters, $p(c)$ and the mean and standard deviation of the Gaussian distributions $f_{j}\left(x_{j} \mid c\right)$ in Equation (1).

Figure 4 about here.

Figure 5 displays the classification tree model which performed second best for dog1, with two observed contexts, Play and Stranger (see the second row in Table 11). Note that three variables are required: Energydiff, Harmmean and Ppj. Thus, if for a given bark, Energydiff $=10$, Harmmean=15 and $\mathrm{Ppj}=0.05$, then the dog is classified as barking at a stranger.

Figure 5 about here.

Figure 6 shows the 100 barks recorded for dog 1 , represented as a point in the 3-D space of three of the five variables selected by the best model, a $k$-nearest neighbors wrapper. Barks in the Play context are colored blue whereas Stranger 
is shaded red. A new bark (an asterisk in the figure) would be classified as the context of its nearest neighbor bark, i.e., Play in this 3-D space, although its nearest nearest neighbor bark should be computed in the 5-D space, also including variables Deviationfreq and Harmmean.

Figure 6 about here.

Table 12 includes the details of the logistic regression model which performed best for dog2, with two observed contexts, Food and Stranger (see the second row in Table 11). This model is built from the five predictor variables in the first column. The regression coefficients $\beta_{j}^{(c)}$ for these variables would be used as in Equation (2) to compute the posterior probability that yields the predicted class.

Table 12 about here.

Individual

A single model for all contexts. Table 13 shows the results of a single model learned from the 800 barks for discriminating among the 8 dogs.

$k$-nearest neighbors wrapper is the best model, as in the three previous classification problems, with an extremely high accuracy, $67.63 \%$, in an 8 multi-class problem. Thus, feature subset selection methods have been proved to produce improvements in model performance.

Table 13 about here.

The true positive rate for each of the classes can be computed from Table 14. Dogs numbers 8,5 and 7 have high true positive rates: $0.77,0.75$ and 0.74 respectively. In contrast, dogs number 6 and 3 have the lowest true positive rates: 0.51 and 0.58 , respectively.

Table 14 about here.

A model per context. More refined context-specific models are built here. By selecting bark sounds from the same context, the corresponding model will classify the individual dog for that context. Thus, a total number of 7 contexts (and their corresponding $12 \times 7$ models) have been considered, where the accuracy of the best model for each context, is shown (see Table 15).

Table 15 about here. 
Note that the model accuracies for identifying dogs have increased to an 80-100\% range compared with the $67.63 \%$ achieved by the global model learned from a database with all the contexts. We now have fewer dogs to be identified, from 2 dogs for the Walk context to 5 dogs for Ball and Fight contexts, whereas the global model had the harder problem of identifying 8 dogs. Although the problem is easier because there are fewer class variable values, barking is expected to be homogeneous in a fixed context, which complicates correct dog identification.

Predictor variables of sex, age, context and individual

The number of selected variables in the best models (see Table 16) represents about $50 \%$ of the 29 initial variables. These numbers were: 12 for Sex, 15 for Age, 16 for Context and 18 for Individual. It is remarkable that some variables, like Ltasm, Ltass, Pitchmint, Pitchslopenojump, and Harmmax were never chosen. On the other hand, the following six variables occur in all four models: Energy, Ltasp, Deviationfreq, Skewness, Pitchq, and Harmmean.

Harmdev appears to be specific for determining dog sex, since it was not selected in the rest of the problems. This also applies to Pitchd, only selected for discriminating dog age and to Pitchmaxt for Individual determination.

Considering the blockwise organization of predictor variables in Table 3, sound energy (first block), source signal (third block) and tonality (fifth block) measurements are sparsely selected compared to a denser selection in the remaining blocks.

Table 16 about here.

\section{Discussion}

This work has empirically demonstrated the usefulness of supervised classification machine learning methods for inferring some characteristics of dogs from the acoustic measurements given by their barks. From the four classification methods considered, $k$-nearest neighbors outperformed naive Bayes, classification trees, and logistic regression. Also, the wrapper feature subset selection method provided significant improvements over a filter selection or no-selection (all variables are kept).

A solution for two prediction problems, sex and age, never previously considered in the literature has been presented. The best of the 12 resulting models in this study was able to predict dog sex in $85.13 \%$ of the cases. The age of 
the dog, categorized as Young, Adult and Old, was inferred correctly in $80.25 \%$ of the cases. An issue to be considered as future work is the prediction of age as a continuous variable, using a kind of regression task.

Determining the context of the dog bark, with seven possible situations, is a more difficult problem than classification by sex and age. However, it was successfully solved for $55.50 \%$ of the bark cases. This is an improvement on the results presented in Molnár et al (2008), where for 6 possible contexts the best model yielded a $43 \%$ success rate. With an accuracy rate of $63 \%$ for classifying three possible contexts, our results are similar to the findings reported by Yin and McCowan (2004). In addition, a model for each of the 8 dogs with two or more different contexts was induced from the barks associated with this specific dog. Thus, a total of $12 \times 8$ models have been considered. For almost all dogs, the $k$-nearest neighbor model was the most successful, although naive Bayes, logistic regression and classification tree models provided the best accuracy results for some dogs. As a tendency the wrapper feature subset selection strategy provided the best results. Model accuracy ranges from $59.80 \%$ to $100 \%$.

The individual identification, a hard classification problem with 8 possible categories, produced up to $67.63 \%$ accuracy in the best model. This result is extremely good when compared to the $52 \%$ reported in Molnár et al (2008) for $14 \mathrm{dogs}$, and the $40 \%$ achieved by Yin and McCowan (2004) for a 10-dog problem. When the dog identification is performed within each context, the accuracies of the best models are in the interval [80.58\%, 100\%].

Recent ethological research on dog barking revealed several features of the most characteristic acoustic communication type of dogs which proved that barks serve as a complex source of information for listeners (Yin and McCowan 2004; Pongrácz et al 2005, 2006). In experiments where human participants evaluated the pre-recorded dog barks, both the context and the possible inner state of the signaling animals were classified with substantial success rates. However, the role of dog barks in dog-dog communication remained (and still remains) somewhat obscure, as there is a shortage of convincing field data for the usage of barks during intraspecific communication of dogs, though see Pongrácz et al (2014) for some positive evidence. The present study provides an alternative approach fort discovering the potential information content encoded in dog barks. If one can prove that dog barks carry consistent cues encoding such features of the caller such as its sex, age, or identity, this can prove indirectly that barks can serve as relevant sources of information to receivers that are able to decipher these types of information.

Previously it was known that dogs can differentiate between individuals and contexts if they hear barks of other dogs in experiments based on the habituation-dishabituation paradigm (Maros et al 2008; Molnár et al 2009). Our 
new results provide some possible details of how such a capacity for recognition might work. If dogs are sensitive to the sex-, age- and identity-specific details of barks, this can serve as an acoustic basis for the cognitive task of discriminating between, or recognition of individuals. Although in dogs sex-related information is mostly (thought to be) transferred via chemical compounds (Goodwin et al 1979), theoretically it would be adaptive if a dog could survey the gender of the other dogs living nearby (or farther) on the basis of hearing their barks as well. Deciphering the age of an individual based on their vocalizations would be also beneficial in a highly social species, where age can be relevant in determining social rank, reproductive status or fighting potential (Mech 1999).

Recognition of the context of barks was the least successful task for our supervised learning methods. Although present methods exceeded the accuracy of both the previously employed machine learning approach (Molnár et al 2008) and the adult human listeners' success rate (Pongrácz et al 2005), this accuracy still lags behind the other variables analyzed in this study. It is also true that human listeners perform almost as successfully when recognizing the context as the computerized models. The reason behind this result may be that the individual variability of dog barks can be considerable especially in particular contexts (such as before the walk, or asking for a toy/food). Another reason for the relatively low success rate of context-recognition may be that while the human listeners received short bark sequences, the computer worked with individual bark sounds. Therefore the interbark interval served as an additional source of information for the humans (Pongrácz et al 2005, 2006), while this parameter was not involved in the computerized analysis. For humans at least, the interbark interval also seemed to be an important source of information when discriminating between individual dogs, as their performance improved with the length of bark sequences they received (Molnár et al 2006).

Supervised classification machine learning methods do not only provide indirect proof about the rich and biologically relevant information content of dog barks, but they also offer a promising tool for applied research, too. For example, evaluating dog behavior has great importance for various organizations, as well as professionals and dog enthusiasts. Recognizing unnecessarily aggressive dogs can be a challenge for the personnel of dog shelters as well as for correspondents of breed clubs and for the experts of legal bodies (Netto and Planta 1997; Serpell and Hsu 2001). Similarly, diagnosing particular behavioral abnormalities that can cause serious welfare issues for dogs, such as separation anxiety, can present a difficult task when the goal is to tell apart 'everyday' and chronic stress reactions in a dog (Overall et al 2001). Behavioral evaluation usually does not cover the qualitative analysis of vocalizations in these 
cases. However, this could be addressed if a reliable and easy to use acoustic analytic software could serve as an aid for behavioral professionals. With such a method, following a rigorous validating protocol, acoustic features indicative of high levels of aggression, fear, distress etc. could be recognized in the subjects' vocalizations.

The limitations of the supervised classification models presented in this paper concern the standard problems with the sample representativeness and the assumptions upon which the models rely. On the other hand the generality of the four methods makes them directly applicable to other species. In addition all the dogs in this study were of the same breed, so our classifiers do not take any advantage of the different patterns expected from the diversity of breeds.

An interesting problem for the near future would be to see whether these methods would work for other breeds or for a mixed breed group. Also, simultaneously classifying the four dog features, sex, age, context and individual, might be of interest. This issue falls into a category of a new problem type called multi-dimensional classification problems (Bielza et al 2011; Borchani et al 2012; Sucar et al 2014), where the dependence between the four class variables is relevant.

Acknowledgements This work has been partially supported by the Spanish Ministry of Economy and Competitiveness, projects Cajal Blue Brain (C080020-09; the Spanish partner of the Blue Brain Project initiative from EPFL) and TIN2013-41592-P, by the János Bolyai Research Scholarship from the Hungarian Academy of Sciences, and co-financed by a grant from OTKA K82020. The authors are thankful to Celeste R. Pongrácz for the English proofreading of this manuscript and to Nikolett Czinege for help in preparing the bark databases.

\section{References}

Acevedo M, Corrada-Bravo C, Corrada-Bravo H, Villanueva-Rivera L, Aide T (2009) Automated classification of bird and amphibian calls using machine learning: A comparison of methods. Ecol Inform 4(4):206-214

Adachi I, Kuwahata H, Fujita K (2007) Dogs recall their owner's face upon hearing the owner's voice. Anim Cogn 10:17-21

Adams M, Law B, Gibson M (2010) Reliable automation of bat call identification for Eastern New South Wales, Australia, using classification trees and AnaScheme software. Acta Chiropterol 12(1):231-245

Armitage D, Ober H (2010) A comparison of supervised learning techniques in the classification of bat echolocation calls. Ecol Inform $5(6): 465-473$

Au W, Andersen L, Roitblat ARH, Nachtigall P (1995) Neural network modeling of a dolphin's sonar discrimination capabilities. J Acoust Soc Am 98:43-50

Bálint A, Faragó T, Dóka A, Miklósi A, Pongrácz P (2013) “Beware, I am big and non-dangerous!” - Playfully growling dogs are perceived larger than their actual size by their canine audience. Appl Anim Behav Sci 148:128-137 
Bielza C, Li G, Larrañaga P (2011) Multi-dimensional classification with Bayesian networks. Int J Approx Reason 52:705-727

Blumstein D, Munos O (2005) Individual, age and sex-specific information is contained in yellow-bellied marmot alarm calls. Anim Behav $69(2): 353-361$

Borchani H, Bielza C, Martínez-Martín P, Larrañaga P (2012) Markov blanket-based approach for learning multi-dimensional Bayesian network classifiers: An application to predict the European Quality of Life-5Dimensions (EQ-5D) from the 39-item Parkinson's Disease Questionnaire (PDQ-39). J Biomed Inform 45:1175-1184

Britzke E, Duchamp J, Murray K, Swihart R, Robbins L (2011) Acoustic identification of bats in the Eastern United States: A comparison of parametric and nonparametric methods. J Wildl Manage 75(3):660-667

Charrier I, Aubin T, Mathevon N (2010) Mother-calf vocal communication in Atlantic walrus: a first field experimental study. Anim Cogn $13(3): 471-482$

Cheng J, Sun Y, Ji L (2010) A call-independent and automatic acoustic system for the individual recognition of animals: A novel model using four passerines. Pattern Recogn 43(11):3846-3852

Chesmore E (2001) Application of time domain signal coding and artificial neural networks to passive acoustical identification of animals. Appl Acoust 62(12):1359-1374

Clemins P (2005) Automatic Classification of Animal Vocalizations. PhD thesis, Marquete University

Cohen J, Fox M (1976) Vocalizations in wild canids and possible effects of domestication. Behav Process 1(77-92)

Coppinger R, Feinstein M (1991) “Hark! Hark! the dogs bark...” and bark and hark. Smithonian 21:119-128

Druzhkova A, Thalmann O, Trifonov V, Leonard J, Vorobieva N, Ovodov N, ASGraphodatsky, Wayne R (2013) Ancient DNA analysis affirms the canid from Altai as a primitive dog. PLoS ONE 8:e57,754

Duda R, Hart P, Stork D (2001) Pattern Classification. John Wiley and Sons

Fant G (1976) Acoustic Theory of Speech Production. Mouton De Gruyter

Faragó T, Pongrácz P, Miklósi A, Huber L, Virányi Z, Range F (2010a) Dogs' expectation about signalers' body size by virtue of their growls. PLoS ONE 5(12):e15,175

Faragó T, Pongrácz P, Range F, Virányi Z, Miklósi A (2010b) "The bone is mine": affective and referential aspects of dog growls. Anim Behav 79(4):917-925

Feddersen-Petersen DU (2000) Vocalization of European wolves (Canis lupus lupus 1.) and various dog breeds (Canis lupus f. fam.). Arch Tierz, Dummerstorf 43(4):387-397

Fix E, Hodges JL (1951) Discriminatory analysis. nonparametric discrimination: Consistency properties. USAF School of Aviation Medicine $4: 261-279$

Frommolt KH, Goltsman M, MacDonald D (2003) Barking foxes, Alopex lagopus: field experiments in individual recognition in a territorial mammal. Anim Behav 65:509-518

Goodwin M, Gooding KM, Regnier F (1979) Sex pheromone in the dog. Science 203:559-561

Gunasekaran S, Revathy K (2011) Automatic recognition and retrieval of wild animal vocalizations. Int J Comput Theor Eng 3(1):136-140 
Hall M (1999) Correlation-based feature selection for machine learning. PhD thesis, Department of Computer Science, University of Waikato

Hall M, Frank E, Holmes G, Pfahringer B, Reutemann P, Witten I (2009) The WEKA data mining software: an update. SIGKDD Explor 11(1):10-18

Hartwig S (2005) Individual acoustic identification as a non-invasive conservation tool: an approach to the conservation of the African wild dog Lycaon pictus (Temminck, 1820). Bioacoustics 15:35-50

Hecht J, Miklósi A, Gácsi M (2012) Behavioral assessment and owner perceptions of behaviors associated with guilt in dogs. Appl Anim Behav Sci 139:134-142

Hunag C, Yang Y, Yang D, Chen Y (2009) Frog classification using machine learning techniques. Expert Syst Appl 36(2):3737-3743

Jain A, Murty MN, Flynn P (1999) Data clustering: A review. ACM Comput Surv 31(3):264-323

Le Cessie S, van Houwelingen JC (1992) Ridge estimators in logistic regression. Appl Stat 41(1):191-201

Liu H, Motoda H (1998) Feature Selection for Knowledge Discovery and Data Mining. Kluwer Academic

Lord K, Feinstein M, Coppinger R (2000) Barking and mobbing. Behav Process 81:358-368

Manser M, Seyfarth R, Cheney D (2002) Suricate alarm calls signal predator class and urgency. Trends Cogn Sci 6(2):55-57

Maros K, Pongrácz P, Bárdos G, Molnár C, Farag T, Miklósi A (2008) Dogs can discriminate barks from different situations. Appl Anim Behav Sci 114:159-167

Mazzini F, Townsend SW, Virányi Z, Range F (2013) Wolf howling is mediated by relationship quality rather than underlying emotional stress. Curr Biol 23:1677-1680

McConnell PB (1990) Acoustic structure and receiver response in domestic dogs, Canis familiaris. Anim Behav 39:897-904

McConnell PB, Baylis JR (1985) Interspecific communication in cooperative herding: acoustic and visual signals from human shepherds and herding dogs. Z Tierpsychol 67:302-382

Mech LD (1999) Alpha status, dominance and division of labor in wolf packs. Can J Zool 77:1196-1203

Meints K, Racca A, Hickey N (2010) Child-dog misunderstandings: children misinterpret dogs' facial expressions. In: Proceedings of the 2nd Canine Science Forum, p 99

Miklósi A, Polgrdi R, Topál J, Csányi V (2000) Intentional behaviour in dog-human communication: an experimental analysis of "showing" behaviour in the dog. Anim Cogn 3:159-166

Minsky M (1961) Steps toward artificial intelligence. T Ins Radio Eng 49:8-30

Molnár C, Pongrácz P, Dóka A, Miklósi A (2006) Can humans discriminate between dogs on the base of the acoustic parameters of barks? Behav Process 73:76-83

Molnár C, Kaplan F, Roy P, Pachet F, Pongrácz P, Dóka A, Moklósi A (2008) Classification of dog barks: a machine learning approach. Anim Cogn 11:389-400

Molnár C, Pongrácz P, Faragó T, Dóka A, Miklósi A (2009) Dogs discriminate between barks: the effect of context and identity of the caller. Behav Process 82(2):198-201

Morton E (1977) On the occurrence and significance of motivation - structural rules in some bird and mammal sounds. Am Nat 111:855-869 
Netto W, Planta D (1997) Behavioural testing for aggression in the domestic dog. Appl Anim Behav Sci 52:243-263

Overall K, Dunham A, Frank D (2001) Frequency of nonspecific clinical signs in dogs with separation anxiety, thunderstorm phobia, and noise phobia, alone or in combination. J Am Vet Med Assoc 219:467-473

Parsons S (2001) Identification of New Zeeland bats (Chalinobus tuberculatus and Mystacina tuberculata) in flight from analysis of echolocation calls by artificial neural networks. J Zool 253(4):447-456

Parsons S, Jones G (2000) Acoustic identification of twelve species of echolocating bat by discriminant function analysis and artificial neural networks. J Exp Biol 203(17):2641-2656

Pongrácz P, Molnár C, Miklósi A, Csányi V (2005) Human listeners are able to classify dog (canis familiaris) barks recorded in different situations. J Comp Psychol 119:136-144

Pongrácz P, Molnár C, Miklósi A (2006) Acoustic parameters of dog barks carry emotional information for humans. Appl Anim Behav Sci $100: 228-240$

Pongrácz P, Molnár C, Miklósi A (2010) Barking in family dogs: an ethological approach. Vet J 183:141-147

Pongrácz P, Szabó E, Kis A, Péter A, Miklósi A (2014) More than noise? Field investigations of intraspecific acoustic communication in dogs (Canis familiaris). Appl Anim Behav Sci in press

Quinlan R (1993) C4.5: Programs for Machine Learning. Morgan Kaufmann

Reid P (2009) Adapting to the human world: dogs' responsiveness to our social cues. Behav Process 80:325-333

Roch M, Soldevilla M, Hoenigman R, Wiggins S, Hidebrand J (2008) Comparison of machine learning techniques for the classification of echolocation clicks from three species of odontocetes. Can Acoust 36(1):41-47

Root-Gutteridge H, Bencsik M, Chebli M, Gentle L, Terrell-Nield C, Bourit A, Yarnell RW (2013) Improving individual identification in captive Eastern grey wolves (Canis lupus lycaon) using the time course of howl amplitudes. Bioacoustics 23(1):39-53

Saeys Y, Inza I, Larrañaga P (2007) A review of feature selection techniques in bioinformatics. Bioinformatics 23(19):2507-2517

Serpell J, Hsu Y (2001) Development and validation of a novel method for evaluating behavior and temperament in guide dogs. Appl Anim Behav Sci 72:347-364

Smith A, Birnie A, Lane K, French J (2009) Production and perception of sex differences in vocalizations of wied's black-tufted-ear marmosets (callithrix kuhlii). Am J Primatol 71:324-332

Stone M (1974) Cross-validatory choice and assessment of statistical predictions. J R Stat Soc B 36(2):111-147

Sucar E, Bielza C, Morales E, Hernandez-Leal P, Zaragoza J, Larrañaga P (2014) Multi-label classification with Bayesian network-based chain classifiers. Pattern Recogn Lett 41:14-22

Taylor A, Reby D, McComb K (2008) Human listeners attend to size information in domestic dog growls. J Acoust Soc Am 123(5):29032909

Taylor A, Reby D, McComb K (2009) Context-related variation in the vocal growling behaviour of the domestic dog (Canis familiaris). Ethology 115(10):905-915

Taylor A, Reby D, McComb K (2010) Size communication in domestic dog, Canis familiaris, growls. Anim Behav 79(1):205-210 
Téglás E, Gergely A, Kupán K, Miklósi A, Topál J (2012) Dogs’ gaze following is tuned to human communicative signals. Curr Biol 22:1-4 Tembrock G (1976) Canid vocalizations. Behav Process 1:57-75

Volodin I, Volodina E, Klenova A, Filatova O (2005) Individual and sexual differences in the calls of the monomorphic white-faced whistling duck dendrocygna viduata. Acta Ornithol 40:43-52

Wan M, Bolger N, Champagne F (2012) Human perception of fear in dogs varies according to experience with dogs. PLoS ONE 7:e51,775 Yeon SC (2007) The vocal communication of canines. J Vet Behav 2:141-144

Yin S (2002) A new perspective on barking in dogs (Canis familiaris). J Comp Psychol 116:189-193

Yin S, McCowan B (2004) Barking in domestic dogs: context specificity and individual identification. Anim Behav 68:343-355

Yovel Y, Au WWL (2010) How can dolphins recognize fish according to their echoes? a statistical analysis of fish echoes. PLoS ONE 5(11):e14,054 
Table 1 Examples of machine learning technique usage from acoustic signals for different species with different aims. Acronyms stand for: $\mathrm{ANN}=$ artificial neural network, $\mathrm{SVM}=$ support vector machine, DFA = discriminant function analysis, GMM = Gaussian mixture model,

$k \mathrm{NN}=k$-nearest neighbor classifier, $\mathrm{HMM}=$ hidden Markov model, $\mathrm{NB}=$ naive Bayes

\begin{tabular}{|c|c|c|c|}
\hline Animal & Aim & Technique & Reference \\
\hline \multirow[t]{2}{*}{ Dolphin } & discriminate cylinder thickness & ANN & Au et al (1995) \\
\hline & classify fish species & SVM, quadratic DFA & Yovel and Au (2010) \\
\hline Odontocete & classify echolocation clicks & GMM, SVM & Roch et al (2008) \\
\hline Walrus & classify barks in mother-calf communication & ANN, DFA & Charrier et al (2010) \\
\hline \multirow[t]{2}{*}{ Frog } & classify species & kNN, SVM & Hunag et al (2009) \\
\hline & & linear DFA, trees, SVM & Acevedo et al (2009) \\
\hline \multirow[t]{2}{*}{ Bird } & classify species & linear DFA, trees, SVM & Acevedo et al (2009) \\
\hline & recognize individuals & GMM & Cheng et al (2010) \\
\hline \multirow[t]{4}{*}{ Bat } & classify species & ANN, DFA & Parsons (2001); Parsons and Jones (2000) \\
\hline & & trees & Adams et al (2010) \\
\hline & & random forests, SVM & Armitage and Ober (2010) \\
\hline & & ANN, DFA, kNN & Britzke et al (2011) \\
\hline Cricket, grasshopper & classify species & ANN & Chesmore (2001) \\
\hline Marmot & classify identity, age and sex & DFA & Blumstein and Munos (2005) \\
\hline Suricate & predict predator type & DFA & Manser et al (2002) \\
\hline \multirow[t]{3}{*}{ African elephant } & classify vocalization type & HMM & Clemins (2005) \\
\hline & classify contexts & HMM & Clemins (2005) \\
\hline & recognize individuals & HMM & Clemins (2005) \\
\hline Female elephant & classify rumbles by oestrous cycle phase & HMM & Clemins (2005) \\
\hline Artic fox & recognize individuals & DFA & Frommolt et al (2003) \\
\hline African wild dog & recognize individuals & DFA & Hartwig (2005) \\
\hline \multirow[t]{2}{*}{ Domestic dog } & classify contexts & DFA & Yin and McCowan (2004) \\
\hline & recognize individuals (breeds) & DFA & Yin and McCowan (2004) \\
\hline \multirow[t]{2}{*}{ Mudi dog } & classify contexts & Gaussian NB & Molnár et al (2008) \\
\hline & recognize individuals & Gaussian NB & Molnár et al (2008) \\
\hline
\end{tabular}


Table 2 Characteristics of the bark data set with seven context categories: Alone, Ball, Fight, Food, Play, Stranger and Walk

\begin{tabular}{|c|c|c|c|c|c|c|c|c|c|c|c|c|}
\hline & & & & & & & Contex & & & & & \\
\hline Number & Dog & Sex & Age & Bouts & Alone & Ball & Fight & Food & Play & Stranger & Walk & Total \\
\hline 1 & Bogyó & male & 1 year & 5 & & & & & 50 & 50 & & 100 \\
\hline 2 & Derüs & female & 2 years & 15 & & & & 50 & & 50 & & 100 \\
\hline 3 & Fecske & female & 2 years & 10 & 25 & 25 & 25 & & & 25 & & 100 \\
\hline 4 & Guba & female & 5 years & 14 & 50 & 50 & & & & & & 100 \\
\hline 5 & Harmat & female & 4 years & 7 & & & 50 & & & 50 & & 100 \\
\hline 6 & Sába & female & 6 years & 7 & & 25 & 25 & 25 & 25 & & & 100 \\
\hline 7 & Ügyes & male & 10 years & 6 & 17 & 17 & 17 & 17 & & 17 & 17 & 102 \\
\hline 8 & Merse & male & 7 years & 6 & 14 & 14 & 14 & 14 & 14 & 14 & 14 & 98 \\
\hline Total & & & & & 106 & 131 & 131 & 106 & 89 & 206 & 31 & 800 \\
\hline
\end{tabular}


Table 3 The 29 acoustic measures extracted from barking recordings

\begin{tabular}{lll}
\hline Name & Description & Variable \\
\hline & Measurements of sound energy & \\
Energy & amount of energy in the sound $\left(\mathrm{Pa}^{2} \cdot s\right)$ & $X_{1}$ \\
Loudness & loudness & $X_{10}$ \\
Ltasm & mean long-term average spectrum $(1 \mathrm{tas})$ & $X_{23}$ \\
Ltass & slope of the ltas & $X_{24}$ \\
Ltasp & local peak height between $1700-3200$ in the 1 tas & $X_{25}$ \\
Ltasd & standard deviation of the ltas & $X_{26}$
\end{tabular}

\section{Measurements of spectral energy}

Banddensity

density of the spectrum between 2000 and $4000 \mathrm{~Hz}$

$X_{2}$

Centerofgravityfreq

average frequency in the spectrum

$X_{3}$

Deviationfreq

standard deviation of the frequency in the spectrum

$X_{4}$

Skewness

skewness of the spectrum

$X_{5}$

Kurtosis

kurtosis of the spectrum

Cmoment

non-normalized skewness of the spectrum

$X_{6}$

$X_{7}$

Energydiff

energy difference between 0-2000 and 2000-6000 Hz bands

Densitydiff

density difference between 0-2000 and 2000-6000 Hz bands

$X_{9}$

\section{Measurements of the source signal}

Pitchm mean fundamental frequency (F0) in Hertz $\quad X$

\section{Pitchmin}

minimum F0

$X_{11}$

Pitchmax

maximum F0

$X_{12}$

Pitchmint

time point of the minimum F0 (s)

$X_{13}$

$X_{14}$

Pitchmaxt

time point of the maximum F0 (s)

$X_{15}$

Pitchd

standard deviation of the F0

$X_{16}$

Pitchq

lower interquantile of the F0

$X_{17}$

Pitchslope

mean absolute slope of the F0

$X_{18}$

Pitchslopenojump

mean slope of the F0 without octave jump

$X_{19}$

\section{Measurements of the voice cycles}

Ppp

number of voice cycles

$X_{20}$

mean number of voice cycles

$X_{21}$

jitter

$X_{22}$

Measures of the tonality

Harmmax

maximum tonality

$X_{27}$

Harmmean

mean tonality

$X_{28}$

standard deviation of the tonality

$X_{29}$ 


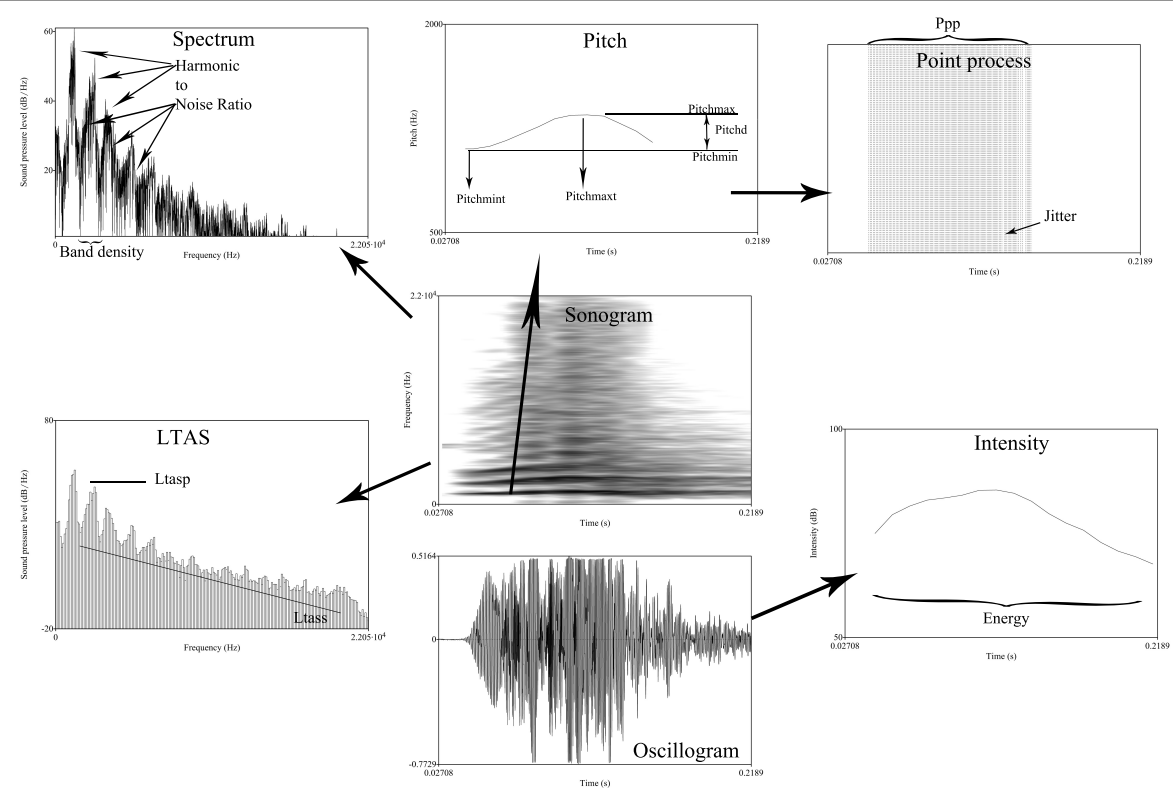

Fig. 1 Main parameters measured for the acoustic analysis using Praat functions. The Oscillogram shows the actual complex waveform of a single bark. The amplitude of the waveform shows the intensity change over time, which is represented here as the Intensity profile. The Energy parameter is the overall energy transferred by the sound over time. Fast Fourier Transformation is used to create a Sonogram which shows the frequency spectrum of the bark over time. Autocorrelation method was applied to extract the fundamental frequency and its profile depicted as the Pitch object. The fundamental frequency is the frequency of opening and closing cycles of the vocal fold, which is represented by the Point process object where every vertical line represents one vocal cycle. This can be used to measure the periodicity of the sound and irregularities in sound production (Jitter). The Spectrum shows the overall power of each frequency component. The Harmonic-to-noise ratio gives the ratio of harmonic spectral components (the upper harmonics of the fundamental frequency) over the irregular, noisy components. Finally, the Long-term Average Spectrum (LTAS) represents the average energy distribution over the frequency spectrum 
1

2

3

4

5

6

7

8

9

10

11

12

13

14

15

16

17

18

19

20

21

22

23

24

25

26

27

28

29

30

31

32

33

34

35

36

37

38

39

40

41

42

43

44

45

46

47

48

49

50

51

52

53

54

55

56

57

58

59

60

61

62

63

64

65
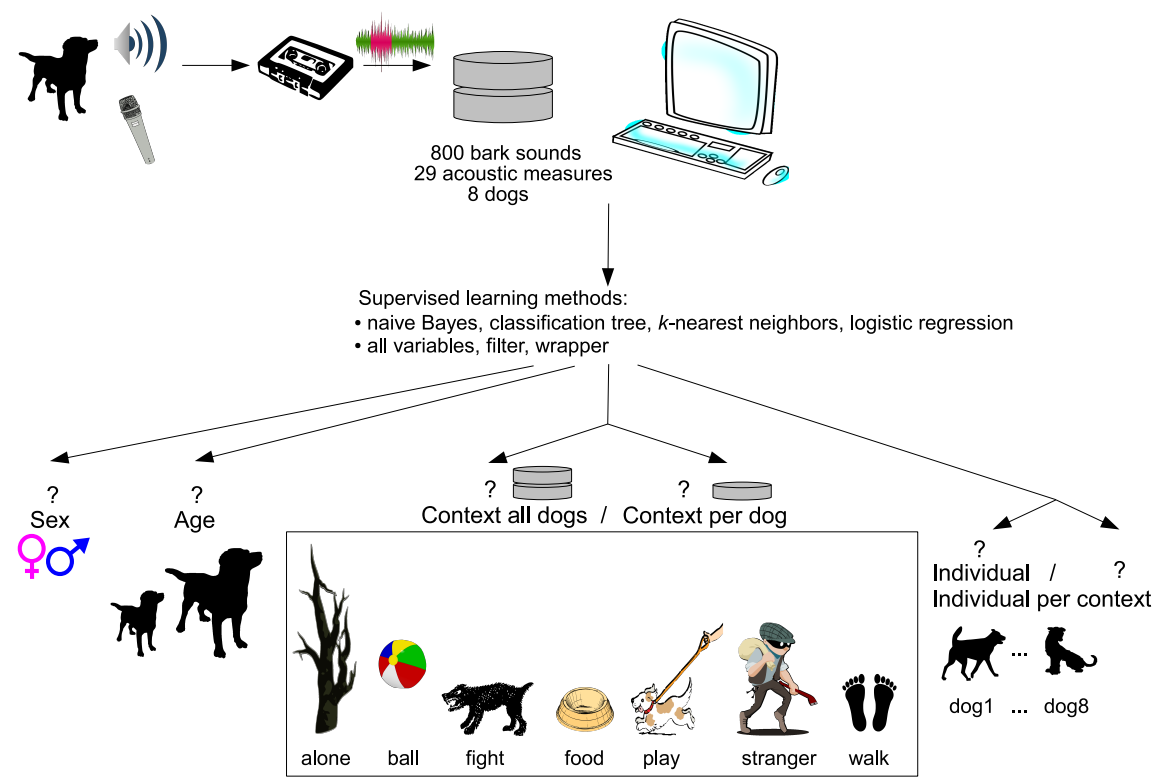

Fig. 2 Diagram of the study: data preprocessing (top) and questions to be answered by machine learning models (bottom) 
Table 4 Raw data in a supervised classification problem: $N$ denotes the number of labeled observations, each of them characterized by $n$ predictor variables, $X_{1}, \ldots, X_{n}$ and the class variable $C . \mathbf{x}^{(N+1)}$ denotes the new observation to be classified by the supervised classification model

\begin{tabular}{l|ccc|c} 
& $X_{1}$ & $\ldots$ & $X_{n}$ & $C$ \\
\hline$\left(\mathbf{x}^{(1)}, c^{(1)}\right)$ & $x_{1}^{(1)}$ & $\ldots$ & $x_{n}^{(1)}$ & $c^{(1)}$ \\
$\left(\mathbf{x}^{(2)}, c^{(2)}\right)$ & $x_{1}^{(2)}$ & $\ldots$ & $x_{n}^{(2)}$ & $c^{(2)}$ \\
$\ldots$ & & $\ldots$ & & $\ldots$ \\
$\left(\mathbf{x}^{(N)}, c^{(N)}\right)$ & $x_{1}^{(N)}$ & $\ldots$ & $x_{n}^{(N)}$ & $c^{(N)}$ \\
$\mathbf{x}^{(N+1)}$ & $x_{1}^{(N+1)}$ & $\ldots$ & $x_{n}^{(N+1)}$ & $?$ \\
\hline
\end{tabular}


1

2

Table 5 Sex prediction. Accuracies of the twelve models: three selection feature methods for each of the four supervised classifiers

\begin{tabular}{lccc}
\hline & all & filter & wrapper \\
\hline Naive Bayes & 71.00 & 71.13 & 77.13 \\
Classification tree & 78.13 & 72.75 & 81.50 \\
$k$-nearest neighbors & 82.00 & 64.25 & $\mathbf{8 5 . 1 3}$ \\
Logistic regression & 76.88 & 70.50 & 78.63 \\
\hline
\end{tabular}


1

Table 6 Sex prediction per dog. Accuracies of the best model in Table 5 for each of the eight dogs. The overall accuracy of this model over the eight dogs is $85.13 \%$

\begin{tabular}{lll}
\hline & Male $76.00 \%$ & Female $90.60 \%$ \\
\hline $\operatorname{dog} 1$ & $73.00 \%$ & - \\
$\operatorname{dog} 2$ & - & $90.00 \%$ \\
$\operatorname{dog} 3$ & - & $87.00 \%$ \\
$\operatorname{dog} 4$ & - & $87.00 \%$ \\
$\operatorname{dog} 5$ & - & $97.00 \%$ \\
$\operatorname{dog} 6$ & - & $92.00 \%$ \\
$\operatorname{dog} 7$ & $79.41 \%$ & - \\
$\operatorname{dog} 8$ & $75.51 \%$ & - \\
\hline
\end{tabular}


Table 7 Age prediction. Accuracies of the twelve models: three selection feature methods for each of the four supervised classifiers (left table). Confusion matrix of the best model: $k$-nearest neighbors wrapper (right table)

\begin{tabular}{lccc}
\hline & all & filter & wrapper \\
\hline Naive Bayes & 68.50 & 65.63 & 71.88 \\
Classification tree & 70.88 & 69.13 & 74.13 \\
$k$-nearest neighbors & 78.63 & 79.13 & $\mathbf{8 0 . 2 5}$ \\
Logistic regression & 75.63 & 73.88 & 76.00 \\
\hline
\end{tabular}

\begin{tabular}{|c|c|c|c|c|}
\hline & & \multicolumn{3}{|c|}{ Predicted class } \\
\hline & & Young & Adult & Old \\
\hline Real & Young & 237 & 55 & 8 \\
\hline \multirow[t]{2}{*}{ class } & Adult & 61 & 328 & 9 \\
\hline & Old & 7 & 18 & 77 \\
\hline
\end{tabular}


1

Table 8 Age prediction per dog. Accuracies of the best model in Table 7 for each of the eight dogs. The overall accuracy of this model over the eight dogs is $80.25 \%$

\begin{tabular}{llll}
\hline & Young $79.00 \%$ & Adult $82.41 \%$ & Old $75.49 \%$ \\
\hline $\operatorname{dog} 1$ & $84.00 \%$ & - & - \\
$\operatorname{dog} 2$ & $74.00 \%$ & - & - \\
$\operatorname{dog} 3$ & $79.00 \%$ & - & - \\
$\operatorname{dog} 4$ & - & $85.00 \%$ & - \\
$\operatorname{dog} 5$ & - & $90.00 \%$ & - \\
$\operatorname{dog} 6$ & - & $66.00 \%$ & - \\
$\operatorname{dog} 7$ & - & - & $75.49 \%$ \\
$\operatorname{dog} 8$ & - & $88.77 \%$ & - \\
\hline
\end{tabular}


Table 9 Context prediction. Accuracies of the twelve models: three selection feature methods for each of the four supervised classifiers (left table). Confusion matrix of the best model: $k$-nearest neighbors wrapper (right table)

\begin{tabular}{lccc}
\hline & all & filter & wrapper \\
\hline Naive Bayes & 41.63 & 42.63 & 47.88 \\
Classification tree & 44.00 & 44.63 & 44.13 \\
$k$-nearest neighbors & 50.88 & 50.75 & $\mathbf{5 5 . 5 0}$ \\
Logistic regression & 49.75 & 47.50 & 50.13 \\
\hline
\end{tabular}

\begin{tabular}{|c|c|c|c|c|c|c|c|c|}
\hline \multirow[b]{3}{*}{ Real } & & \multicolumn{7}{|c|}{ Predicted class } \\
\hline & & Alone & Ball & Fight & Food & Play & Stranger & Walk \\
\hline & Alone & 46 & 15 & 7 & 17 & 6 & 14 & 1 \\
\hline \multirow[t]{6}{*}{ class } & Ball & 11 & 64 & 5 & 22 & 5 & 23 & 1 \\
\hline & Fight & 8 & 4 & 100 & 3 & 4 & 11 & 1 \\
\hline & Food & 7 & 20 & 2 & 55 & 3 & 15 & 4 \\
\hline & Play & 8 & 8 & 2 & 10 & 44 & 11 & 6 \\
\hline & Stranger & 12 & 24 & 5 & 26 & 13 & 124 & 2 \\
\hline & Walk & 0 & 3 & 4 & 5 & 6 & 2 & 11 \\
\hline
\end{tabular}


Table 10 Context prediction per dog. Accuracies of the best model in Table 9 for each of the eight dogs. The overall accuracy of this model over the eight dogs is $55.50 \%$

\begin{tabular}{|c|c|c|c|c|c|c|c|}
\hline & Alone $43.40 \%$ & Ball $48.85 \%$ & Fight $76.34 \%$ & Food $51.89 \%$ & Play $49.44 \%$ & Stranger $60.19 \%$ & Walk $35.48 \%$ \\
\hline $\operatorname{dog} 1$ & - & - & - & - & $76.00 \%$ & $68.00 \%$ & - \\
\hline $\operatorname{dog} 2$ & - & - & - & $64.00 \%$ & - & $54.00 \%$ & - \\
\hline $\operatorname{dog} 3$ & $52.00 \%$ & $44.00 \%$ & $56.00 \%$ & - & - & $60.00 \%$ & - \\
\hline $\operatorname{dog} 4$ & $44.00 \%$ & $60.00 \%$ & - & - & - & - & - \\
\hline $\operatorname{dog} 5$ & - & - & $98.00 \%$ & - & - & $70.00 \%$ & - \\
\hline $\operatorname{dog} 6$ & - & $36.00 \%$ & $76.00 \%$ & $44.00 \%$ & $12.00 \%$ & - & - \\
\hline $\operatorname{dog} 7$ & $52.94 \%$ & $29.41 \%$ & $52.94 \%$ & $17.65 \%$ & - & $35.29 \%$ & $41.18 \%$ \\
\hline $\operatorname{dog} 8$ & $14.29 \%$ & $64.29 \%$ & $57.14 \%$ & $64.29 \%$ & $21.43 \%$ & $50.00 \%$ & $14.29 \%$ \\
\hline
\end{tabular}


Table 11 Context discrimination: A model per dog. Summary of the best models, accuracies and corresponding contexts for each dog. Dogs are organized by number of contexts and then by model accuracy

\begin{tabular}{llll}
\hline Dog & Model & Accuracy & Context \\
\hline $\operatorname{dog} 5$ & naive Bayes wrapper & & \\
& $k$-nearest neighbors wrapper & 100.00 & Fight $\cdot$ Stranger \\
$\operatorname{dog} 1$ & $k$-nearest neighbors wrapper & 97.00 & Play $\cdot$ Stranger \\
$\operatorname{dog} 2$ & logistic regression wrapper & 86.00 & Food $\cdot$ Stranger \\
$\operatorname{dog} 4$ & naive Bayes wrapper & 78.00 & Alone $\cdot$ Ball \\
$\operatorname{dog} 3$ & $k$-nearest neighbors wrapper & 74.00 & Alone $\cdot$ Ball $\cdot$ Fight $\cdot$ Stranger \\
$\operatorname{dog} 6$ & logistic regression wrapper & 73.00 & Ball $\cdot$ Fight $\cdot$ Food $\cdot$ Play \\
$\operatorname{dog} 7$ & naive Bayes wrapper & 59.80 & Alone $\cdot$ Ball $\cdot$ Fight $\cdot$ Food $\cdot$ Stranger $\cdot$ Walk \\
$\operatorname{dog} 8$ & $k$-nearest neighbors wrapper & 66.98 & Alone $\cdot$ Ball $\cdot$ Fight $\cdot$ Food $\cdot$ Play $\cdot$ Stranger $\cdot$ Walk \\
\hline
\end{tabular}


1

2

3

4

5

6

7

8

9

10

11

12

13

14

15

16

17

18

19

20

21

22

23

24

25

26

27

28

29

30

31

32

33

34

35

36

37

38

39

40

41

42

43

44

45

46

47

48

49

50

51

52

53

54

55

56

57

58

59

60

61

62

63

64

65

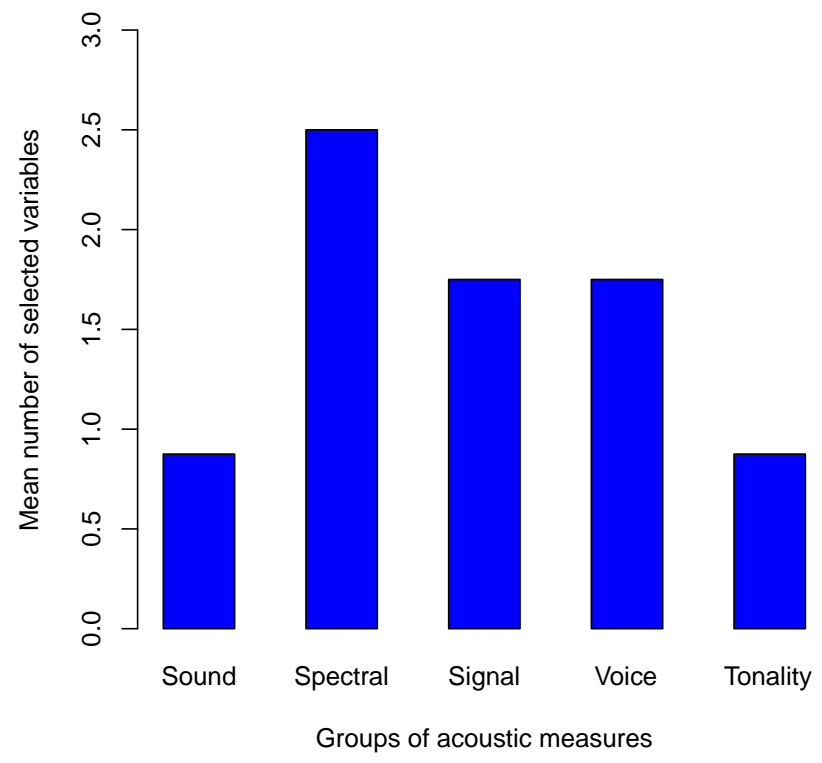

Fig. 3 Mean number of variables ( $Y$-axis) selected by the best models per dog when predicting contexts (listed in Table 11), for each of the five groups of acoustic measures ( $X$-axis): sound energy, spectral energy, source signal, voice cycles and tonality. Each of these groups of acoustic measures contain $6,8,9,3$ and 4 variables respectively 
1

2

3

4

5

6

7

8

9

10

11

12

13

14

15

16

17

18

19

20

21

22

23

24

25

26

27

28

29

30

31

32

33

34

35

36

37

38

39

40

41

42

43

44

45

46

47

48

49

50

51

52

53

54

55

56

57

58

59

60

61

62

63

64

65

Fig. 4 Example of a naive Bayes wrapper model. It corresponds to the best model for context classification in dog5 
1

2

3

4

5

6

7

8

9

10

11

12

13

14

15

16

17

18

19

20

21

22

23

24

25

26

27

28

29

30

31

32

33

34

35

36

37

38

39

40

41

42

43

44

45

46

47

48

49

50

51

52

53

54

55

56

57

58

59

60

61

62

63

64

65

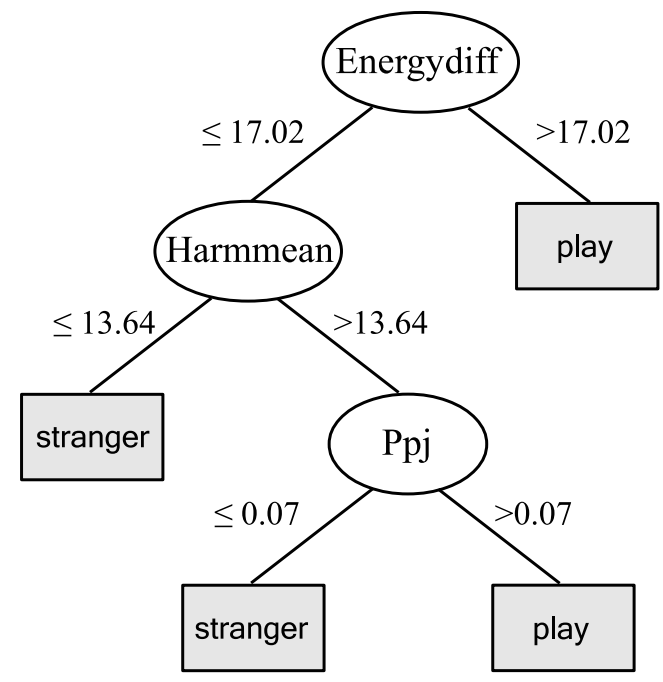

Fig. 5 Example of a classification tree wrapper model. It corresponds to the second best model for context classification in dog1 
1

2

3

4

5

6

7

8

9

10

11

12

13

14

15

16

17

18

19

20

21

22

23

24

25

26

27

28

29

30

31

32

33

34

35

36

37

38

39

40

41

42

43

44

45

46

47

48

49

50

51

52

53

54

55

56

57

58

59

60

61

62

63

64

65

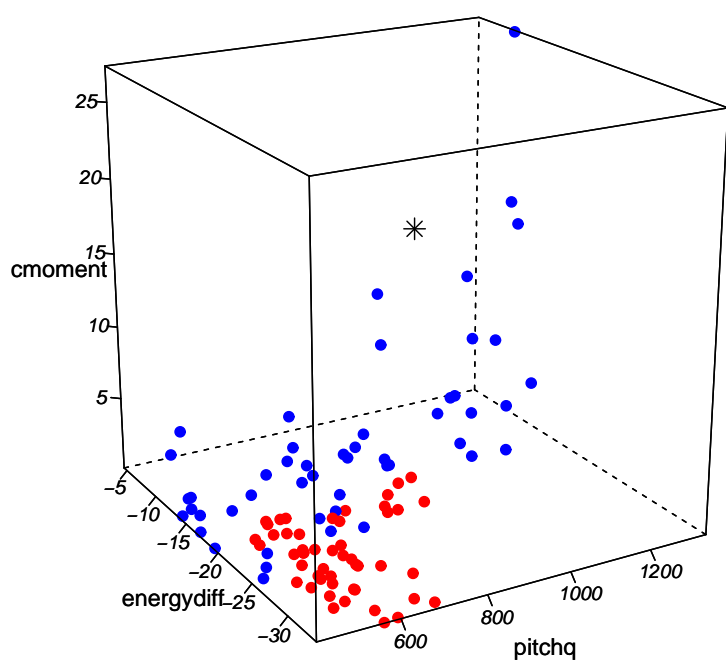

play stranger

Fig. 6 Example of a $k$-nearest neighbors wrapper model. It corresponds to the best model for context classification in dog1 (Cmoment scale is divided by $10^{9}$ ). Classification of a hypothetical bark (asterisk) 
1

2

3

4

5

6

7

8

9

10

11

12

13

14

15

16

17

18

19

20

21

22

23

24

25

26

27

28

29

30

31

32

33

34

35

36

37

38

39

40

41

42

43

44

45

46

47

48

49

50

51

52

53

54

55

56

57

58

59

60

61

62

63

64

65

Table 12 Example of parameter values of a logistic regression model. It corresponds to the best model for context classification in dog2

\begin{tabular}{lr}
\hline Variable $X_{j}$ & $\beta_{j}^{(\text {Food })}$ \\
\hline Kurtosis & -0.0008 \\
Pitchd & -0.0002 \\
Pitchslope & 0.0001 \\
Ppp & -0.0143 \\
Ppm & -7424.9241 \\
Intercept $\left(\beta_{0}\right)$ & 31.5997 \\
\hline
\end{tabular}


1

2

Table 13 Individual prediction. Accuracies of the twelve models: three selection feature methods for each of the four supervised classifiers

\begin{tabular}{llll}
\hline & all & filter & wrapper \\
\hline Naive Bayes & 54.50 & 55.63 & 63.00 \\
Classification tree & 53.13 & 51.37 & 56.37 \\
$k$-nearest neighbors & 63.87 & 58.62 & $\mathbf{6 7 . 6 3}$ \\
Logistic regression & 63.00 & 61.75 & 65.75 \\
\hline
\end{tabular}


Table 14 Confusion matrix for the best model, $k$-nearest neighbors wrapper, identifying individual dogs

\begin{tabular}{cc|cccccccc} 
& & \multicolumn{7}{|c}{ Predicted class } \\
& Dog & 1 & 2 & 3 & 4 & 5 & 6 & 7 & 8 \\
\hline \multirow{4}{*}{ Real } & 3 & 68 & 10 & 8 & 5 & 0 & 5 & 3 & 1 \\
& 2 & 6 & 71 & 5 & 1 & 5 & 8 & 2 & 2 \\
class & 4 & 7 & 3 & 4 & 67 & 2 & 9 & 2 & 6 \\
& 5 & 1 & 6 & 3 & 2 & 75 & 7 & 6 & 0 \\
& 6 & 5 & 12 & 11 & 6 & 6 & 51 & 9 & 0 \\
& 7 & 2 & 3 & 2 & 5 & 5 & 10 & 74 & 1 \\
& 8 & 1 & 4 & 1 & 8 & 1 & 4 & 2 & 77 \\
\hline
\end{tabular}


Table 15 Summary of the results of classifying individuals by context. $k$-nearest neighbors performed best for Alone, Ball, Play and Walk contexts, naive Bayes for Fight and Walk, logistic regression for Stranger and Walk, and classification trees for Food context. All best models corresponded to a wrapper feature subset selection strategy

\begin{tabular}{llll}
\hline Context & No. Barks & No. Dogs & Accuracy \\
\hline Alone & 106 & 4 & 94.34 \\
Ball & 131 & 5 & 80.92 \\
Fight & 131 & 5 & 88.55 \\
Food & 106 & 4 & 87.74 \\
Play & 89 & 3 & 97.75 \\
Stranger & 206 & 5 & 80.58 \\
Walk & 31 & 2 & 100.00 \\
\hline
\end{tabular}


Table 16 Predictor variables of sex, age, context and individual classification problems from the best model, $k$-nearest neighbors wrapper. The accuracies of these four models are: $85.13 \%$ for Sex classification (Table 5), $80.25 \%$ for Age prediction (Table 7 ), 55.50\% for Context categorization (Table 9), and 67.63\% for Individual recognition (Table 13)

\begin{tabular}{|c|c|c|c|c|c|}
\hline Var & Name & Sex & Age & Context & Individual \\
\hline$X_{1}$ & Energy & $\mathrm{x}$ & $\mathrm{x}$ & $\mathrm{x}$ & $\mathrm{x}$ \\
\hline$X_{10}$ & Loudness & & & $\mathrm{x}$ & $\mathrm{x}$ \\
\hline$X_{23}$ & Ltasm & & & & \\
\hline$X_{24}$ & Ltass & & & & \\
\hline$X_{25}$ & Ltasp & $\mathrm{x}$ & $\mathrm{x}$ & $\mathrm{x}$ & $\mathrm{x}$ \\
\hline$X_{26}$ & Ltasd & $\mathrm{x}$ & $\mathrm{x}$ & & \\
\hline$X_{2}$ & Bandensity & & $\mathrm{x}$ & $\mathrm{x}$ & $\mathrm{x}$ \\
\hline$X_{3}$ & Centerofgravityfreq & $\mathrm{x}$ & & $\mathrm{x}$ & $\mathrm{x}$ \\
\hline$X_{4}$ & Deviationfreq & $\mathrm{x}$ & $\mathrm{x}$ & $\mathrm{x}$ & $\mathrm{x}$ \\
\hline$X_{5}$ & Skewness & $\mathrm{x}$ & $\mathrm{x}$ & $\mathrm{x}$ & $\mathrm{x}$ \\
\hline$X_{6}$ & Kurtosis & & $\mathrm{x}$ & $\mathrm{x}$ & $\mathrm{x}$ \\
\hline$X_{7}$ & Cmoment & & $\mathrm{x}$ & $\mathrm{x}$ & $\mathrm{x}$ \\
\hline$X_{8}$ & Energydiff & & $\mathrm{x}$ & $\mathrm{x}$ & $\mathrm{x}$ \\
\hline$X_{9}$ & Densitydiff & & $\mathrm{x}$ & & \\
\hline$X_{11}$ & Pitchm & $\mathrm{x}$ & & & $\mathrm{x}$ \\
\hline$X_{12}$ & Pitchmin & & & & $\mathrm{x}$ \\
\hline$X_{13}$ & Pitchmax & & & $\mathrm{x}$ & $\mathrm{x}$ \\
\hline$X_{14}$ & Pitchmint & & & & \\
\hline$X_{15}$ & Pitchmaxt & & & & $\mathrm{x}$ \\
\hline$X_{16}$ & Pitchd & & $\mathrm{x}$ & & \\
\hline$X_{17}$ & Pitchq & $\mathrm{x}$ & $\mathrm{x}$ & $\mathrm{x}$ & $\mathrm{x}$ \\
\hline$X_{18}$ & Pitchslope & & & & $\mathrm{x}$ \\
\hline$X_{19}$ & Pitchslopenojump & & & & \\
\hline$X_{20}$ & Ppp & $\mathrm{x}$ & $\mathrm{x}$ & $\mathrm{x}$ & \\
\hline$X_{21}$ & Ppm & & $\mathrm{x}$ & $\mathrm{x}$ & $\mathrm{x}$ \\
\hline$X_{22}$ & Ppj & $\mathrm{x}$ & & $\mathrm{x}$ & \\
\hline$X_{27}$ & Harmmax & & & & \\
\hline$X_{28}$ & Harmmean & $\mathrm{x}$ & $\mathrm{x}$ & $\mathrm{x}$ & $\mathrm{x}$ \\
\hline$X_{29}$ & Harmdev & $\mathrm{x}$ & & & \\
\hline
\end{tabular}


Table 1: Examples of machine learning technique usage from acoustic signals for different species with different aims. Acronyms stand for: $\mathrm{ANN}=$ artificial neural network, $\mathrm{SVM}=$ support vector machine, $\mathrm{DFA}=$ discriminant function analysis, $\mathrm{GMM}=$ Gaussian mixture model, $k \mathrm{NN}=k$-nearest neighbor classifier, $\mathrm{HMM}=$ hidden Markov model, $\mathrm{NB}=$ naive Bayes

Table 2: Characteristics of the bark data set with seven context categories: Alone, Ball, Fight, Food, Play, Stranger and Walk

Table 3: The 29 acoustic measures extracted from barking recordings

Table 4: Raw data in a supervised classification problem: $N$ denotes the number of labeled observations, each of them characterized by $n$ predictor variables, $X_{1}, \ldots, X_{n}$ and the class variable $C . \mathbf{x}^{(N+1)}$ denotes the new observation to be classified by the supervised classification model

Table 5: Sex prediction. Accuracies of the twelve models: three selection feature methods for each of the four supervised classifiers

Table 6: Sex prediction per dog. Accuracies of the best model in Table 5 for each of the eight dogs. The overall accuracy of this model over the eight dogs is $85.13 \%$

Table 7: Age prediction. Accuracies of the twelve models: three selection feature methods for each of the four supervised classifiers (left table). Confusion matrix of the best model: $k$-nearest neighbors wrapper (right table)

Table 8: Age prediction per dog. Accuracies of the best model in Table 7 for each of the eight dogs. The overall accuracy of this model over the eight dogs is $80.25 \%$ 
Table 9: Context prediction. Accuracies of the twelve models: three selection feature methods for each of the four supervised classifiers (left table). Confusion matrix of the best model: $k$-nearest neighbors wrapper (right table)

Table 10: Context prediction per dog. Accuracies of the best model in Table 9 for each of the eight dogs. The overall accuracy of this model over the eight dogs is $55.50 \%$

Table 11: Context discrimination: A model per dog. Summary of the best models, accuracies and corresponding contexts for each dog. Dogs are organized by number of contexts and then by model accuracy

Table 12: Example of parameter values of a logistic regression model. It corresponds to the best model for context classification in $\operatorname{dog} 2$

Table 13: Individual prediction. Accuracies of the twelve models: three selection feature methods for each of the four supervised classifiers

Table 14: Confusion matrix for the best model, $k$-nearest neighbors wrapper, identifying individual dogs

Table 15: Summary of the results of classifying individuals by context. $k$-nearest neighbors performed best for Alone, Ball, Play and Walk contexts, naive Bayes for Fight and Walk, logistic regression for Stranger and Walk, and classification trees for Food context. All best models corresponded to a wrapper feature subset selection strategy

Table 16: Predictor variables of sex, age, context and individual classification problems from the best model, $k$ nearest neighbors wrapper. The accuracies of these four models are: $85.13 \%$ for Sex classification (Table 5), 80.25\% for Age prediction (Table 7), 55.50\% for Context categorization (Table 9), and 67.63\% for Individual recognition (Table 13) 
1

2

3

4

5

9

10

11

12

13

14

15

16

17

18

19

20

21

22

23

24

25

26

27

28

29

30

31

32

33

34

35

36

37

38

39

40

41

42

43

44

45

46

47

48

49

50

51

52

53

54

55

56

57

58

59

60

61

62

63

64

65 


\section{Figure Captions}

Figure 1: Main parameters measured for the acoustic analysis using Praat functions. The Oscillogram shows the actual complex waveform of a single bark. The amplitude of the waveform shows the intensity change over time, which is represented here as the Intensity profile. The Energy parameter is the overall energy transferred by the sound over time. Fast Fourier Transformation is used to create a Sonogram which shows the frequency spectrum of the bark over time. Autocorrelation method was applied to extract the fundamental frequency and its profile depicted as the Pitch object. The fundamental frequency is the frequency of opening and closing cycles of the vocal fold, which is represented by the Point process object where every vertical line represents one vocal cycle. This can be used to measure the periodicity of the sound and irregularities in sound production (Jitter). The Spectrum shows the overall power of each frequency component. The Harmonic-to-noise ratio gives the ratio of harmonic spectral components (the upper harmonics of the fundamental frequency) over the irregular, noisy components. Finally, the Long-term Average Spectrum (LTAS) represents the average energy distribution over the frequency spectrum

Figure 2: Diagram of the study: data preprocessing (top) and questions to be answered by machine learning models (bottom)

Figure 3: Mean number of variables selected by the best models for each of the five groups of acoustic measures: sound energy, spectral energy, source signal, voice cycles and tonality. Each of these groups of acoustic measures contain $6,8,9,3$ and 4 variables respectively

Figure 4: Example of a naive Bayes wrapper model. It corresponds to the best model for context classification in $\operatorname{dog} 5$

Figure 5: Example of a classification tree wrapper model. It corresponds to the second best model for context classification in $\operatorname{dog} 1$ 
Figure 6: Example of a $k$-nearest neighbors wrapper model. It corresponds to the best model for context classification in $\operatorname{dog} 1$ (Cmoment scale is divided by $\left.10^{9}\right)$. Classification of a hypothetical bark (asterisk) 


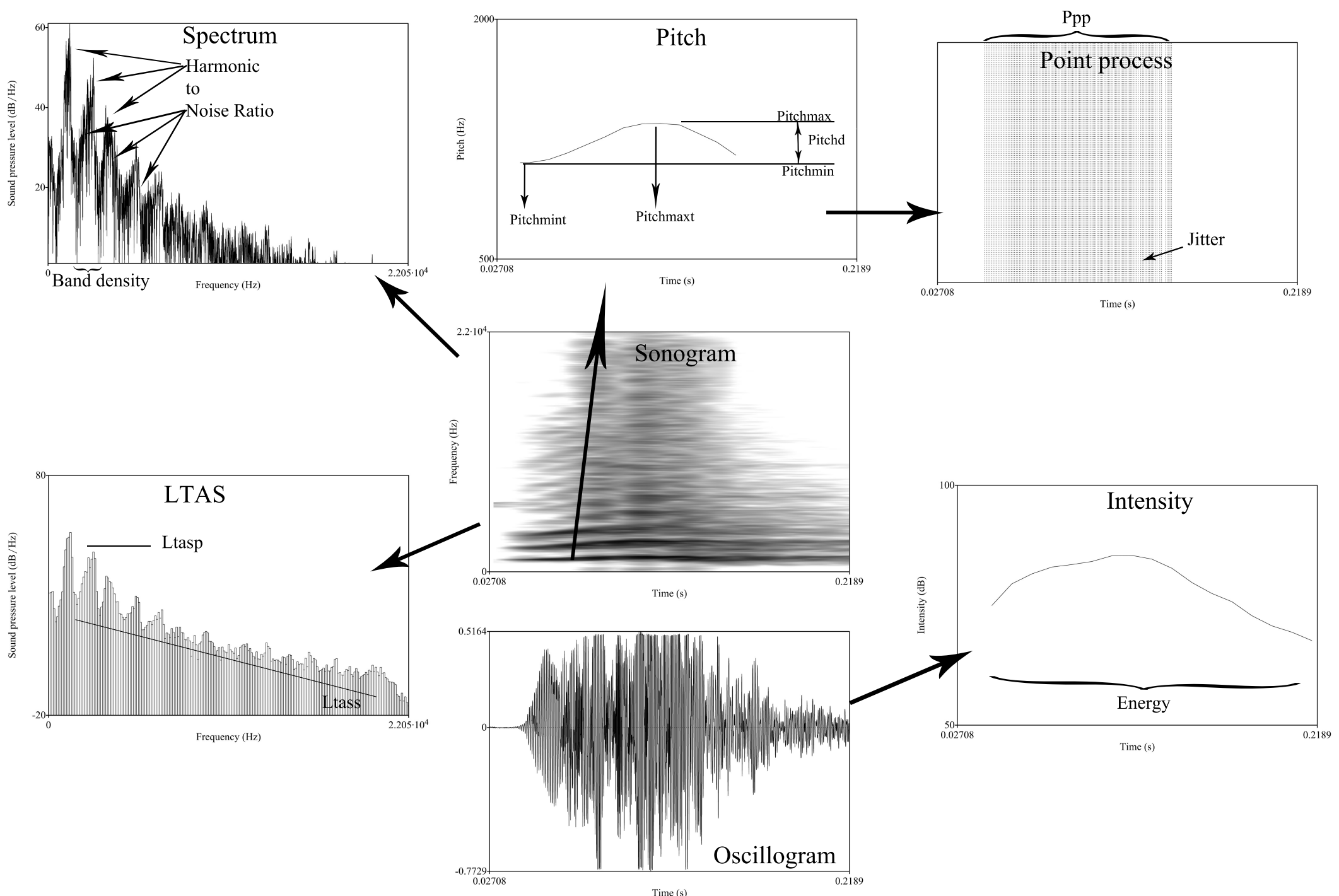




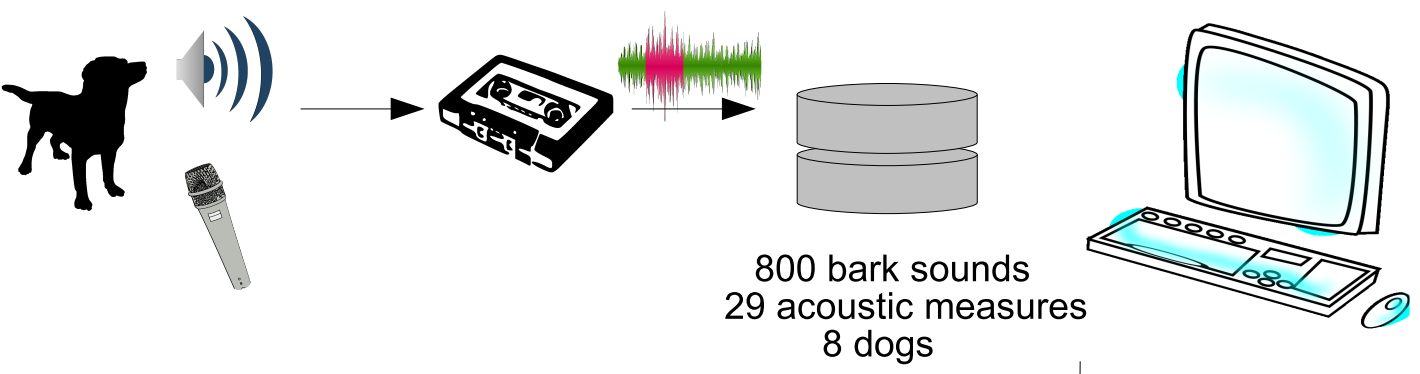

\section{$\checkmark$}

Supervised learning methods:

- naive Bayes, classification tree, $k$-nearest neighbors, logistic regression

- all variables, filter, wrapper
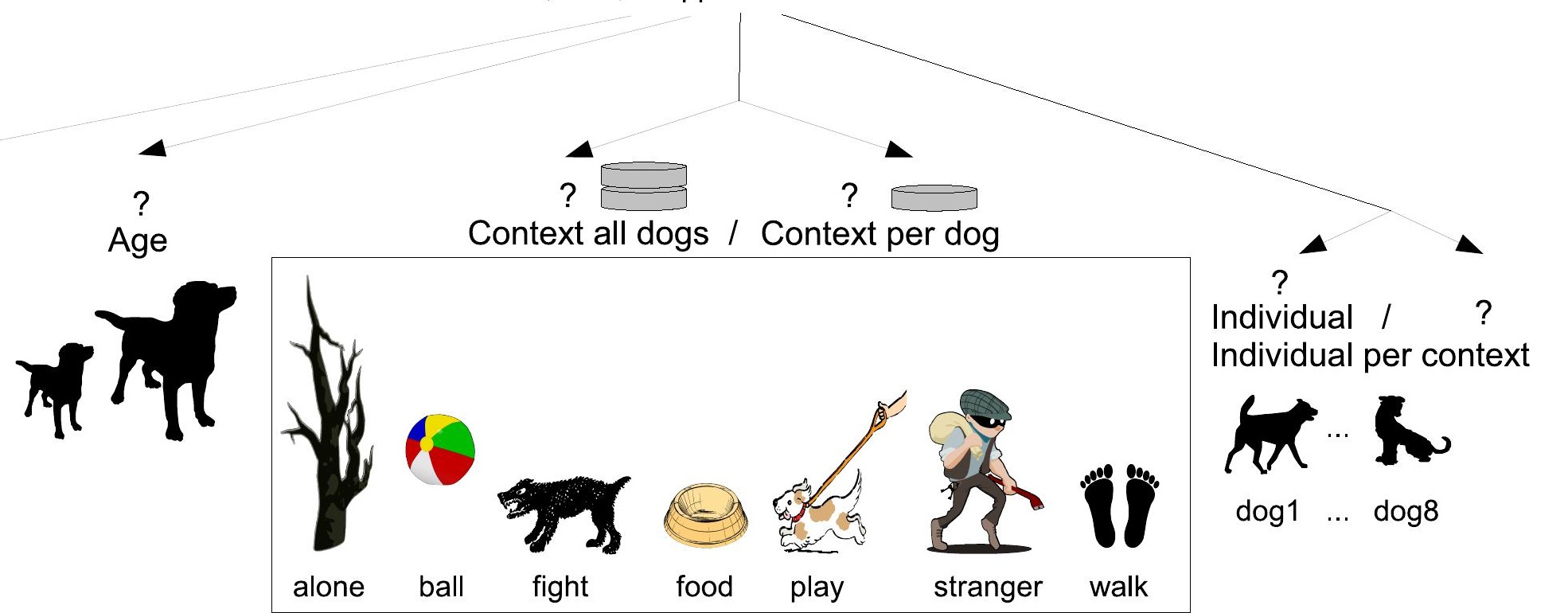

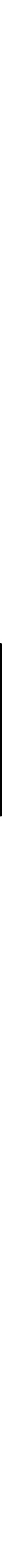

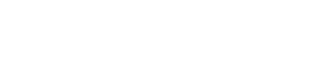
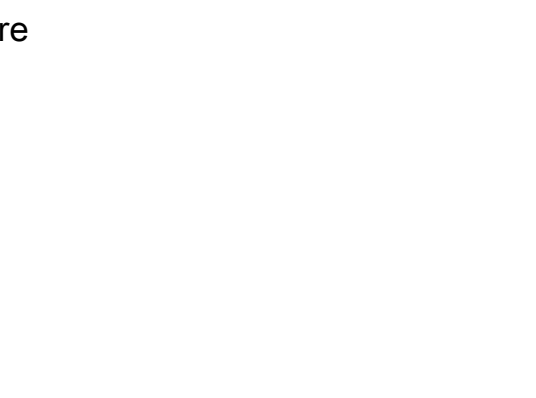


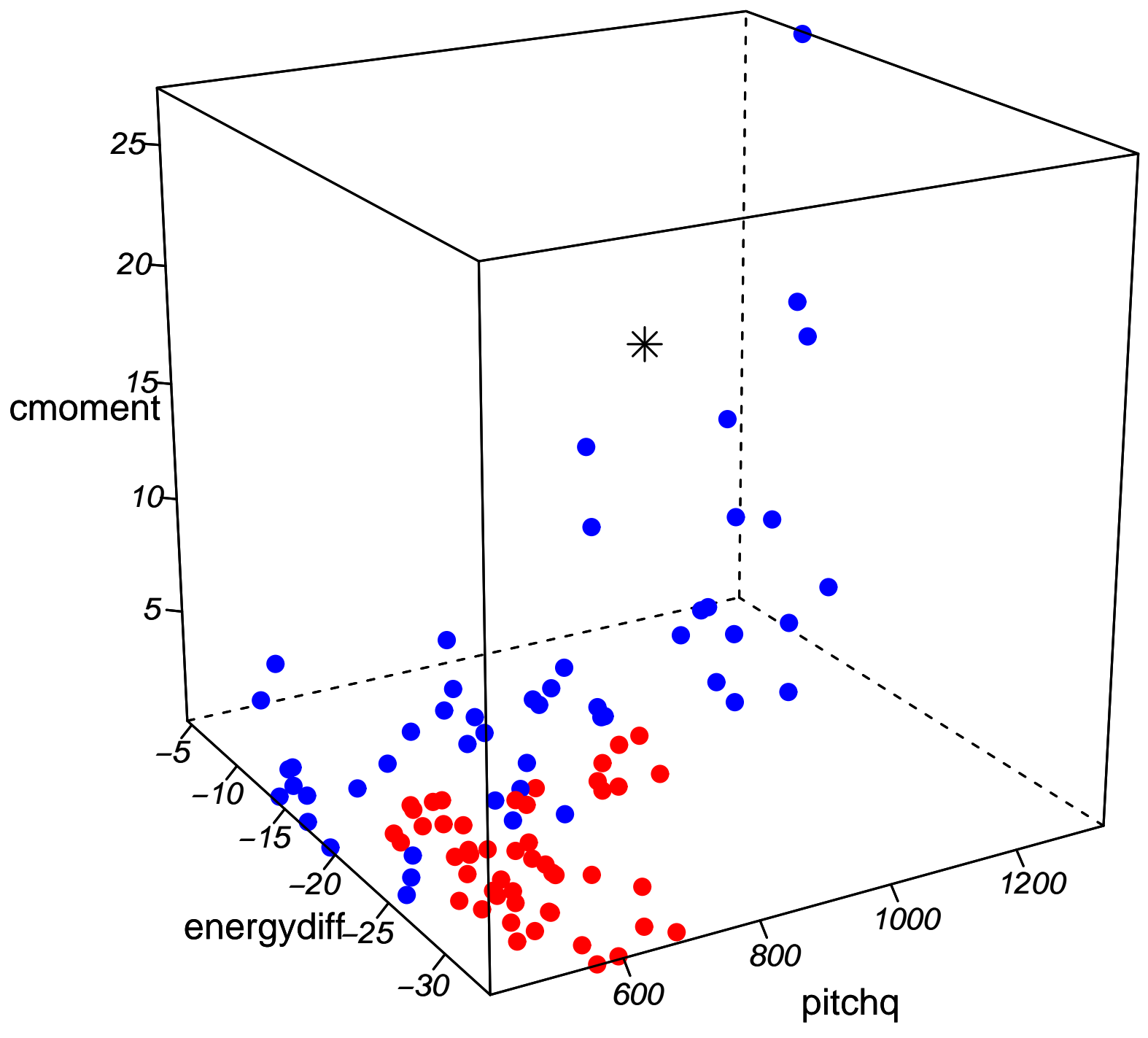

play 
Supplementary Material
Click here to download Supplementary Material: supplementary-material.pdf Click here to dow (1)

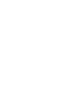

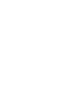
. (1) (1)

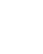
(1) (1) (1) $\sqrt{3}$

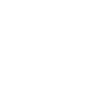
列

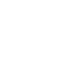
.

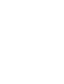

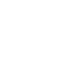
更 更 更 更

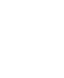
更

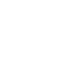

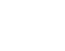

